\title{
Robustness Analysis of Integrated LPV-FDI Filters and LTI-FTC System for a Transport Aircraft
}

\author{
Thuan H. Khong \\ NASA Langley Research Center \\ Jong-Yeob Shin \\ National Institute of Aerospace
}

\begin{abstract}
This paper proposes an analysis framework for robustness analysis of a nonlinear dynamics system that can be represented by a polynomial linear parameter varying (PLPV) system with constant bounded uncertainty. The proposed analysis framework contains three key tools: 1) a function substitution method which can convert a nonlinear system in polynomial form into a PLPV system, 2) a matrix-based linear fractional transformation (LFT) modeling approach, which can convert a PLPV system into an LFT system with the delta block that includes key uncertainty and scheduling parameters, 3) $\mu$-analysis, which is a well known robust analysis tool for linear systems. The proposed analysis framework is applied to evaluating the performance of the LPV-fault detection and isolation (FDI) filters of the closedloop system of a transport aircraft in the presence of unmodeled actuator dynamics and sensor gain uncertainty. The robustness analysis results are compared with nonlinear time simulations.
\end{abstract}

\section{Nomenclature}

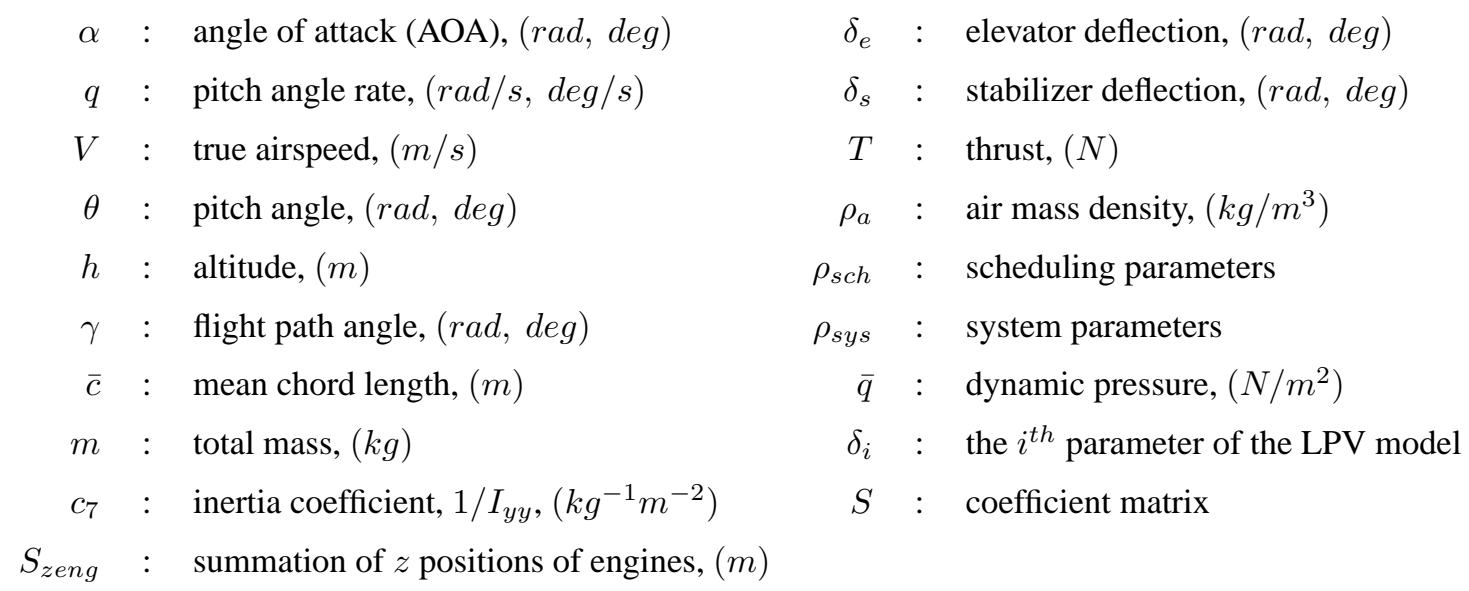




\section{Aerodynamic coefficients}

$\begin{array}{ll}C_{D_{\text {Mach }}} & : \text { drag coefficient at fixed mach number } \\ C_{L} & : \text { total lift coefficient } \\ C_{L_{\text {basic }}} & : \text { lift coefficient for the rigid airplane at zero stabilizer angle } \\ C_{m} & : \text { pitch moment coefficient } \\ C_{m_{\text {basic }}} & : \text { pitch moment coefficient for the rigid airplane at zero stabilizer angle } \\ K_{\alpha} & : \text { effective factor of the elevator }\end{array}$

\section{Introduction}

In general, robustness of a nonlinear system is an open problem. Due to the large diversity of nonlinear models and computational difficulties, there is no unified analysis method for all possible classes of nonlinear dynamics. This paper is concerned with robustness analysis of a class of nonlinear systems described by polynomial functions. This class of nonlinear systems can be represented by a polynomial linear parameter varying (PLPV) system, whose system matrices are polynomial functions of scheduling parameters. Conventionally, stability and performance analysis of an LPV system has been formulated into a linear matrix inequality (LMI) feasibility problem or a performance index minimization problem with LMI constraints, which represent a Lyapunov function type of analysis $[1,9,12,13,21,28]$. For LMI approaches, an LPV system can be described as a set of convex sets [10] or as a set of linear systems defined at grid points over the scheduling parameter spaces $[22,28]$. Robustness analysis of an LPV system, which can capture a nonlinear system, unmodeled dynamics, real parameter uncertainty, and time-varying uncertainty, can be included in an LMI optimization $[1,12,19]$ with scaling matrices. In this paper, instead of using a convex set defined by vertex systems or a set of linear systems defined at grid points, nonlinear system variations due to scheduling parameter change are converted into an LFT model with a structured delta block, which contains scheduling parameters. The key difference between an LMI approach and the proposed analysis framework in this paper is that the nonlinear dynamics are converted to an LFT model with the structured delta block, which can cover all possible variations of scheduling parameters. The variations defined by the structured block are treated as an uncertainty set in the robustness analysis framework. This analysis framework is applied to the robustness analysis of the LPV-FDI filters integrated with the closed-loop system of a transport aircraft.

Robustness analysis of an integrated fault tolerant control (FTC) system with fault detection and isolation (FDI) filters has been of interest to the NASA Aviation Safety Program (NASA AvSP) [23]. Specifically, a fault tolerant control law prevents fault propagation and reduces closed-loop performance degradation due to faults. It makes the detection of faults by FDI filters more difficult in the integrated system. In the validation and verification process of a fault tolerant flight system, the robust performance analysis of FDI filters is important before implementing these into real systems. In [11,29], robust FDI filters are designed for an linear time invariant (LTI) system under feedback control using LMI techniques to maximize fault sensitivity performance and to simultaneously minimize the influence of unknown inputs, which represent dynamic effects of uncertainty. The singular value $\mu$ analysis method has been used to analyze robustness of FDI filters on the LTI systems in [11]. In [23], robustness analysis of an integrated system with an FTC law and FDI filters was presented using $\mu$ analysis. The nonlinear longitudinal motion of a transport aircraft was converted into a PLPV system using the function substitution method. A function substitution method has been introduced in [27] to convert a nonlinear system into a quasi-LPV model. The function substitution method has been modified in $[8,22]$ to minimize variations of decision variables over the scheduling parameter space using linear optimization. The method has been sucessfully applied to develop an LPV model from a nonlinear system including 
look-up table aerodynamic coefficients [22] or polynominal fitted aerodynamic coefficients [23]. For nonlinear dynamics described in polynominal form, the function substitution method is automatically coded to provide a PLPV model [24] using the symbolic toolbox in MATLAB. In this paper, a PLPV system is constructed from the combined model of the LPV model of a tranpsort aircraft and its LPV-FDI filters since the scheduling parameters for the aircraft model and its filters are the same.

In [23], the nonlinear dynamics of a transport aircraft were converted into a PLPV system without considering the LPV-FDI filters. The PLPV system was converted into an LFT model with the delta block, which contained all dynamic purturbations due to changes in flight condition. In [23], robustness analysis of the integrated system with an FTC law and LTI-FDI filters in the presence of unmodeled actuator dynamics was presented. In this paper, a robustness analysis framework of polynomial LPV-FDI filters is introduced. Robustness analysis of the transport aircraft (a Boeing 747-100/200 aircraft) is performed using three key tools: 1) function substitution method, 2) LFT modeling approach, and 3) $\mu$ analysis tool. The previously developed robustness analysis framework in [23] is extended to analyze the performance of a class of nonlinear multi-variable PLPV dynamics systems. The robustness analysis framework is illustrated by analyzing the robust performance of the integrated LPV-FDI filters and FTC system for a Boeing 747-100/200 aircraft.

The organization of the paper is as follows. The matrix-based LFT modeling approach [5,7] is discussed in Section 2. In Section 3, the analysis framework for a nonlinear multi-variable polynomial system is presented. In Section 4, the analysis method is introduced and illustrated via an example of robustness analysis of two LPV-FDI filters for a Boeing 747-100/200 nonlinear longitudinal dynamics model. The nonlinear longitudinal dynamics equations of the airplane, the actuator models, the LTI-FTC controller, and the FDI filters are from [8], [18], and [26], and are presented in Sections 4.1 to 4.3. The nonlinear equations of longitudinal dynamics of the airplane and of the LPV-FDI filters are rewritten as polynomial functions of the deviations of the states and inputs from their trim values. In Section 4.4, the polynomial models of the airplane and the filters are combined and converted into an LFT model with the parameters in the diagonal "uncertainty" block being velocity and angle of attack. The resulting LFT model is then connected with the controller, the linear actuator models, and the weighting functions. The closed-loop interconnected system and the weighting functions are presented in Section 4.5. Results of the robustness analysis and nonlinear simulation are presented in Section 4.6. Section 5 presents concluding remarks.

\section{Preliminary Theory Background}

There are a few methods $[16,17]$ of obtaining an LFT model from a multi-variable PLPV model. One of those methods is the matrix-based LFT modeling approach [5-7,14]. The approach converts multi-variable PLPV models to their equivalent LFT models. Some of the key concepts of the matrix-based LFT modeling approach are summarized in this section because this approach is used in this paper, and also because these key concepts give insights into the equivalence between a multi-variable PLPV model and its LFT model.

Consider

$$
\left[\begin{array}{l}
\dot{x} \\
y
\end{array}\right]=\left[\begin{array}{ll}
A\left(\delta_{1}, \ldots, \delta_{i}, \ldots, \delta_{n}\right) & B\left(\delta_{1}, \ldots, \delta_{i}, \ldots, \delta_{n}\right) \\
C\left(\delta_{1}, \ldots, \delta_{i}, \ldots, \delta_{n}\right) & D\left(\delta_{1}, \ldots, \delta_{i}, \ldots, \delta_{n}\right)
\end{array}\right]\left[\begin{array}{l}
x \\
u
\end{array}\right]=S\left(\rho_{\text {sys }}\right)\left[\begin{array}{l}
x \\
u
\end{array}\right]
$$

where $\delta_{i}$ contains a constant nominal component $\delta_{i_{o}}$ and a bounded varying component $\delta_{i_{\Delta}}$

$$
\delta_{i}=\delta_{i_{o}}+\delta_{i_{\Delta}}
$$

$\rho_{\text {sys }}$ represents the vector of system parameters $\delta_{1}, \ldots, \delta_{i}, \ldots, \delta_{n}$, and $A\left(\rho_{\text {sys }}\right), B\left(\rho_{\text {sys }}\right), C\left(\rho_{\text {sys }}\right)$, and $D\left(\rho_{\text {sys }}\right)$ are multi-variable polynomial matrix functions of $\delta_{i}$. It is apparent that $S\left(\rho_{\text {sys }}\right)$ is also a multi-variable polynomial matrix 
function of $\delta_{i}$. If the coefficient matrix of the term $\delta_{1}^{\eta_{1}} \ldots \delta_{i}^{\eta_{i}} \ldots \delta_{n}^{\eta_{n}}$, where $\eta_{i}$ is the exponent of $\delta_{i}$, is denoted by

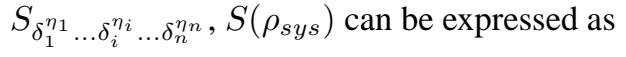

$$
S\left(\rho_{\text {sys }}\right)=\sum_{\eta_{1}=0}^{m_{1}} \ldots \sum_{\eta_{i}=0}^{m_{i}} \cdots \sum_{\eta_{n}=0}^{m_{n}}\left(S_{\delta_{1}^{\eta_{1}} \ldots \delta_{i}^{\eta_{i}} \ldots \delta_{n}^{\eta_{n}}} \prod_{i=1}^{n} \delta_{i}^{\eta_{i}}\right)
$$

where $m_{i}$, which is finite, is the maximum degree of $\delta_{i}$. The coefficient matrix $S_{\delta_{1}^{0} \ldots \delta_{i}^{0} \ldots \delta_{n}^{0}}$, denoted as $S_{o}$, is the nominal component of the LPV model.

Consider a sketch of an LFT model in Fig. 1. The matrix $\Delta\left(\rho_{\text {sys }}\right)$ contains the system variation, and can be represented as follows

$$
\Delta\left(\rho_{s y s}\right)=\operatorname{diag}\left[\delta_{1} I_{k_{1}}, \ldots, \delta_{i} I_{k_{i}}, \ldots, \delta_{n} I_{k_{n}}\right]
$$

The structures of $P, R, L, Q$ matrices, suggested by [5], are

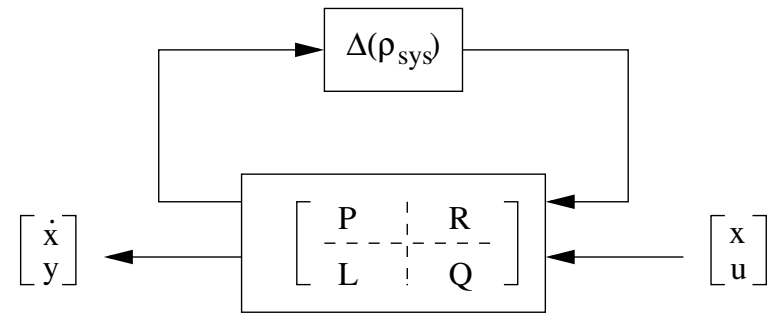

Figure 1: Block diagram of LFT model.

$$
\begin{gathered}
P=\left[\begin{array}{ccccc}
P_{11} & P_{12} & P_{13} & \ldots & P_{1 n} \\
0_{k_{2} \times k_{1}} & P_{22} & P_{23} & \ldots & P_{2 n} \\
0_{k_{3} \times k_{1}} & 0_{k_{3} \times k_{2}} & P_{33} & \ldots & P_{3 n} \\
\ldots & \ldots & \ldots & \ddots & \vdots \\
0_{k_{n} \times k_{1}} & 0_{k_{n} \times k_{2}} & 0_{k_{n} \times k_{3}} & \ldots & P_{n n}
\end{array}\right], \quad R=\left[\begin{array}{c}
R_{1} \\
\vdots \\
R_{n}
\end{array}\right] \\
L=\left[L_{1} \ldots L_{n}\right], \quad Q=S_{o} .
\end{gathered}
$$

The LFT equation associated with Fig. 1 is given below

$$
S\left(\rho_{\text {sys }}\right)=S_{\Delta}\left(\rho_{\text {sys }}\right)+S_{o}=L(I-\Delta P)^{-1} \Delta R+Q=L \Delta(I-P \Delta)^{-1} R+Q .
$$

If $(I-\Delta P)^{-1}$ (or $(I-P \Delta)^{-1}$ ) is replaced with a Taylor series expansion, the right hand side of Eq. (7) has the form of the right hand side of Eq. (3). With the given coefficient matrices, matrix theory is used to solve Eq. (7) for $P, R$, $L$, and $Q$ such that the following conditions are satisfied: 1) $P$ is a nilpotent matrix so that the Taylor series expansion of the matrix inversion is finite, and 2) $L_{i}, P_{i j}, R_{i}(i=1, \ldots, n, j=1, \ldots, n)$ are found such that the expansion of $L(I-\Delta P)^{-1} \Delta R+Q$ matches with the given coefficient matrices term by term. The size of the identity matrix $I_{k_{i}}$ associated with $\delta_{i}$ does not have to be known; it is found when the equation is solved. The dimension of the system variation block $\Delta\left(\rho_{\text {sys }}\right)$ is $\sum_{i=1}^{n} k_{i}$. Details of how Eq. (7) is solved are beyond the scope of this paper and can be 
found in [5-7]. Other LFT modeling methods are based on a symbolic approach [3,17]. It is noted that the LFT model equivalent to the given LPV model is not unique. The system variation block of the LFT model found by any of the three methods does not necessarily have the smallest dimension possible.

The parameters in $\Delta$ are normalized to be consistent with the convention of $\mu$ analysis. The normalization of the parameters can be done before or after the LFT model is obtained. Before normalization, the range of parameter $\delta_{i}$ is $\left[\delta_{i_{\min }}, \delta_{i_{\max }}\right]$, assuming $\delta_{i}$ is bounded. After normalization, the range of $\delta_{i}$ is $[-1,1]$. Although it seems trivial to mention the fact that $-1 \leq$ normalized $\delta_{i} \leq 1$, this remark helps explain the results of $\mu$ analysis in Section 4 . The maximum singular value of the normalized variation block $(\bar{\sigma}(\Delta))$ can be thought of as "distance" from the nominal of the normalized parameters; thus, the $\mu$ value is the inverse of the maximum "distance" from the nominal of the normalized parameters, which still satisfies the performance of the system.

In brief, this section presents some key concepts in the matrix-based LFT modeling approach to transform a given multi-variable PLPV model into an LFT model. A multi-variable PLPV model and its equivalent LFT model, therefore, are two mathematical ways of expressing the same model. Thus, $\mu$ analysis on the LFT model yields another approach to analyzing PLPV models.

\section{Analysis Framework}

Consider a nonlinear closed-loop system, that has the form of Eq. (1), where $A, B, C$, and $D$ are polynomial matrix functions of $\rho_{\text {sys }}$. A robustness analysis problem is defined as "Can the closed-loop system achieve a pre-defined performance level in the presence of constant but unknown uncertainty such as unmodeled actuator dynamics and sensor gain uncertainty over the operating envelope?" In the analysis framework, there are three steps:

1. The nonlinear system is converted into a PLPV system using a function substitution method [8, 23, 24].

2. The PLPV system is converted into an LFT model with a structured variation block of the system scheduling parameters using the matrix-based LFT modeling approach described in the previous section.

3. The augmented closed-loop system with performance weighting matrices, actuator unmodeled dynamics, and sensor gain uncertainty in the form of an LFT model is analyzed using the $\mu$ analysis tool.

Robust performance analysis of LPV-FDI filters using this analysis framework are presented in the next section.

\section{Robust Performance Analysis of LPV-FDI Filters of Boeing 747-100/200}

The FDI filters of the airplane are considered robust in this paper if they can detect faults over the considered flight envelope of $[150,250] \mathrm{m} / \mathrm{s}$ for velocity and $[-2,8]$ degrees for angle of attack at the considered altitude of $7000 \mathrm{~m}$. The variation block of the system parameters can be thought of as a structured uncertainty block, so the word "robust" here agrees with its conventional meaning. The robustness of the integrated LPV-FDI filters and closed-loop FTC model in the conventional sense (i.e., in the presence of model uncertainty) is also considered in Section 4.6. For simplicity, it is assumed that the airplane model and the actuator models have no uncertainty unless mentioned otherwise. Fig. 2 shows the block diagram of the closed-loop control of Boeing 747-100/200 and its FDI filters. In the sketch, $\rho_{\text {sch }}$ represents the scheduling parameters. The nonlinear closed-loop FTC model and the LPV-FDI filters are taken from $[8,18,26]$. 


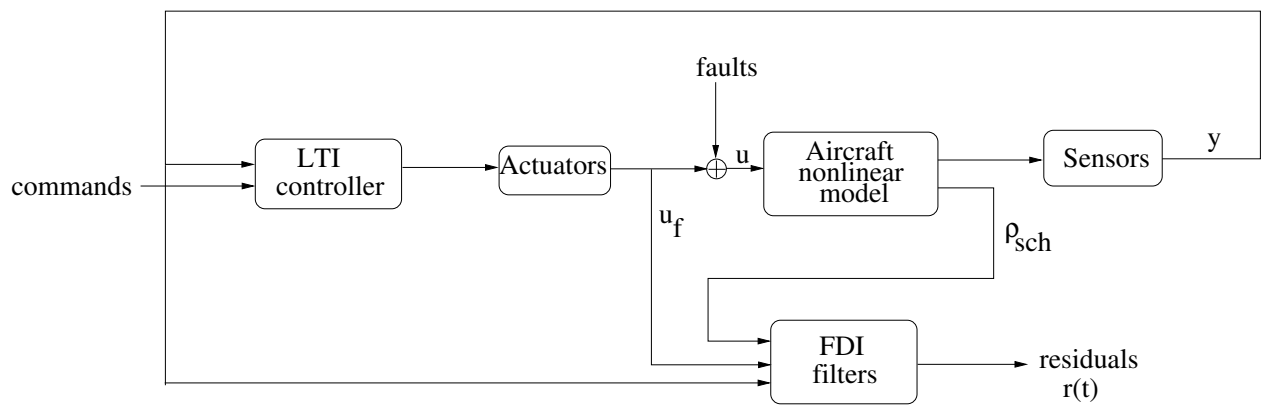

Figure 2: Block diagram of closed-loop system of a Boeing 747-100/200 aircraft and its FDI filters.

\subsection{LTI controller and actuators}

The LTI controller of the system in Fig. 2 is an $H_{\infty}$ controller, which is from [18]. The controller has eighteen states, six inputs, and three outputs. The inputs of the controller are $\gamma_{c m d}-\tilde{\gamma}, \dot{V} / g, \tilde{\theta}, q, \tilde{V}, V_{c m d}$, where the subscript $\mathrm{cmd}$ stands for command and (.) denotes the deviation of a state from its trim value. The outputs of the controller are signals to the elevator actuators, stabilizer actuators, and the engines (thrust actuators). Following [26], the transfer function of the elevator is $\frac{37}{s+37}$, and the transfer function for thrust is $\frac{0.5}{s+0.5}$, The stabilizer input to the airplane will not be used in the robust performance analysis of the FDI filters because the stabilizers are assumed to stay at their trim positions. The stabilizer transfer function is, therefore, not included.

\subsection{Nonlinear model of Boeing 747-100/200}

The nonlinear longitudinal dynamics model of Boeing 747-100/200 are from [8].

$$
\begin{gathered}
\dot{\alpha}=\left[1-\frac{\bar{q} S \bar{c}}{2 m V^{2}}\left(1.45-1.8 x_{c g}\right) \frac{d C_{L}}{d q}\right] q+\left[-\frac{\bar{q} S}{m V} K_{\alpha} \frac{d C_{L}}{d \delta_{e}}\right] \delta_{e}+\left[-\frac{4}{m V}(\sin \alpha+0.0436 \cos \alpha)\right] T+\mathcal{A} \\
\mathcal{A}=\frac{1}{V}(\sin \alpha \sin \theta+\cos \alpha \cos \theta) g-\frac{\bar{q} S}{m V} C_{L_{b a s i c}} \\
\dot{q}=\quad \frac{c_{7} \bar{q} S \bar{c}^{2}}{2 V}\left[\frac{d C_{m}}{d q}-\frac{1}{\bar{c}}\left(1.45-1.8 x_{c . g .}\right) \frac{d C_{L}}{d_{q}}\left(\cos \alpha \bar{x}_{c . g .}+\sin \alpha \bar{z}_{c . g .}\right)\right] q \\
+c_{7} \bar{q} S \bar{c} K_{\alpha}\left[\frac{d C_{m}}{d \delta_{e}}-\frac{1}{\bar{c}} \frac{d C_{L}}{d \delta_{e}}\left(\cos \alpha \bar{x}_{c . g .}+\sin \alpha \bar{z}_{c . g .}\right)\right] \delta_{e} \\
+c_{7} \bar{q} S \bar{c} K_{\alpha} \frac{d C_{m}}{d \delta_{s}} \delta_{s}+c_{7} S_{z e n g} T+\mathcal{Q} \\
\mathcal{Q}=c_{7} \bar{q} S \bar{c} C_{m_{\text {basic }}}+c_{7} \bar{q} S\left[C_{D_{M a c h}}\left(\cos \alpha \bar{z}_{c . g .}-\sin \alpha \bar{x}_{c . g .}\right)-C_{L_{b a s i c}}\left(\cos \alpha \bar{x}_{c . g .}+\sin \alpha \bar{z}_{c . g .}\right)\right] \\
\dot{V}_{t}=\frac{4}{m}(\cos \alpha-0.0436 \sin \alpha) T+\mathcal{V} \\
\mathcal{V}=(\sin \alpha \cos \theta-\cos \alpha \sin \theta) g-\frac{\bar{q} S}{m} C_{D_{M a c h}}
\end{gathered}
$$




$$
\begin{gathered}
\dot{\theta}=q \\
\dot{h}=(\cos \alpha \sin \theta-\sin \alpha \cos \theta) V
\end{gathered}
$$

The altitude of the airplane is weakly coupled with other states via air mass density, and speed of sound, which affects the aerodynamic coefficients. It is assumed that the air mass density and speed of sound are constant in the neighborhood of the considered altitude. With these assumptions, the state $h$ can be removed without affecting other states in Eqs. (8-15). It is noted that state removal is problem-dependent.

Before the above highly nonlinear equations are approximated as polynomial functions of velocity and angle of attack, several sub-problems must be solved. The aerodynamic coefficients are tabulated in look-up tables [18], and thus must be polynomial fitted. The polynomial fitted aerodynamic coefficients, using the method in $[15,20]$, in the velocity range of $[150,250] \mathrm{m} / \mathrm{s}$, and the angle of attack range of $[-2,8]$ degrees at the altitude of $7000 \mathrm{~m}$ are

$$
\begin{gathered}
C_{D_{M a c h}}=\kappa_{20} \alpha^{2}+\kappa_{10} \alpha+\kappa_{01} V+\kappa_{00} \\
\frac{d C_{L}}{d \delta_{e}}=\tau_{02} V^{2}+\tau_{01} V+\tau_{00} \\
C_{L_{\text {basic }}}=\eta_{10} \alpha+\eta_{01} V+\eta_{00} \\
C_{m_{\text {basic }}}=\xi_{20} \alpha^{2}+\xi_{10} \alpha+\xi_{01} V+\xi_{00} \\
\frac{d C_{m}}{d \delta_{e}}=\zeta_{02} V^{2}+\zeta_{01} V+\zeta_{00}
\end{gathered}
$$

where

$$
\begin{array}{llll}
\kappa_{20}=3.2653 & \kappa_{10}=3.4772 \times 10^{-2} & \kappa_{01}=4.4526 \times 10^{-5} & \kappa_{00}=9.9258 \times 10^{-3} \\
\tau_{02}=-1.3593 \times 10^{-7} & \tau_{01}=3.9396 \times 10^{-5} & \tau_{00}=3.5418 \times 10^{-3} & \\
\eta_{10}=5.1806 & \eta_{01}=1.2560 \times 10^{-3} & \eta_{00}=-4.0826 \times 10^{-3} & \\
\xi_{20}=2.3923 & \xi_{10}=-1.4614 & \xi_{01}=-3.2036 \times 10^{-4} & \xi_{00}=0.1264 \\
\zeta_{02}=4.3535 \times 10^{-7} & \zeta_{01}=-1.1644 \times 10^{-4} & \zeta_{00}=-1.7626 \times 10^{-2} &
\end{array}
$$

Other aerodynamic coefficients are assumed to be constant [18], $\frac{d C_{L}}{d q}=4.1, \frac{d C_{m}}{d q}=-17.6, \frac{d C_{m}}{d \delta_{s}}=-2.8$. The aerodynamic coefficients are polynomial functions of $V$ and $\alpha$. The nonlinear equations Eqs. (8-15) with tabulated aerodynamic coefficients are called high fidelity nonlinear (HNL) equations; those equations with polynomiial fitted aerodynamic coefficients are called low fidelity nonlinear (LNL) equations; the LNL equations, after all the nonlinear terms are expressed as polynomials of the states, and the state $h$ is removed, are called polynomial (PN) equations. Later, HNL, LNL, and PN will be applied to the equations of the FDI filters with the same connotation. Let $x_{t}$ be the trim value of the state $x$. Let $\tilde{x}$ be the deviation of the state $x$ from its trim value $\left(\tilde{x}=x-x_{t}\right)$. The trim values of the states and inputs of the nonlinear model with polynomial fitted aerodynamic coefficients (LNL model) are $\left[\alpha_{t}, q_{t}, V_{t}, \theta_{t}, h_{t}\right]=[1.05 \mathrm{deg}, 0 \mathrm{deg} / \mathrm{s}, 227.02 \mathrm{~m} / \mathrm{s}, 1.05 \mathrm{deg}, 7000 \mathrm{~m}]$, and $\left[\delta_{e_{t}}, \delta_{s_{t}}, T_{t}\right]=$ [0.163 deg, $0.590 \mathrm{deg}, 42291 \mathrm{~N}]$. For comparison, the trim conditions of the high fidelity nonlinear (HNL) model of Boeing 747-100/200 are $\left[\alpha_{t H N L}, q_{t H N L}, V_{t H N L}, V_{t H N L}, \theta_{t H N L}, h_{t H N L}\right]=[1.25 \mathrm{deg}, 0 \mathrm{deg} / \mathrm{s}, 227.42 \mathrm{~m} / \mathrm{s}$, $1.25 \mathrm{deg}, 7000 \mathrm{~m}]$, and $\left[\delta_{e_{t H N L}}, \delta_{s_{t H N L}}, T_{t H N L}\right]=[-3.19 \mathrm{deg}, 1.99 \mathrm{deg}, 42291 \mathrm{~N}]$. The trim conditions of the LNL model will be used for the analysis. The quantity $1 / V$ is approximated by its first order Taylor series expansion, $1 / V_{t}-\left(1 / V_{t}^{2}\right) \tilde{V}$. It is assumed that $\tilde{\alpha}, \tilde{\theta}$ are small angles, thus $\sin \alpha \simeq \alpha, \sin \theta \simeq \theta, \cos \alpha \simeq 1, \cos \theta \simeq 1$. After the 

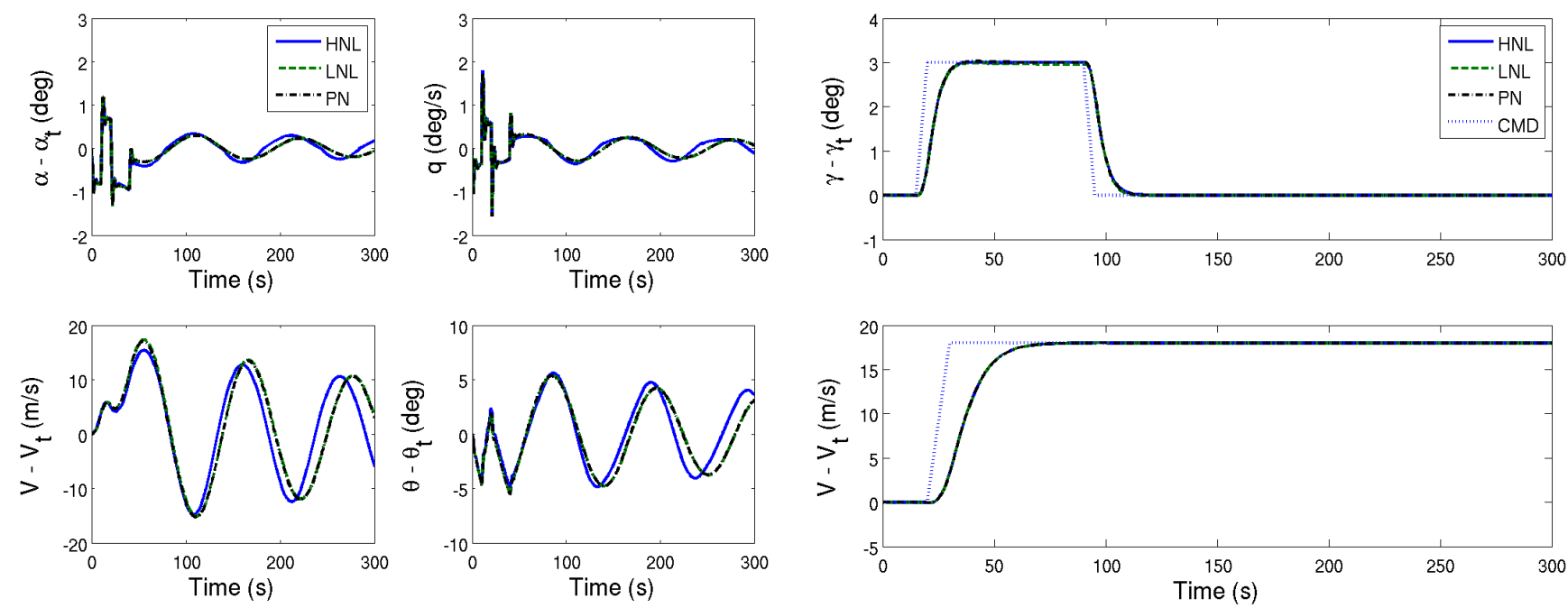

Figure 3: Time responses of the open-loop system under elevator input at the altitude of $7000 \mathrm{~m}$.

Figure 4: Time responses of the closed-loop system under $\gamma$ - and velocity-commands at the altitude of $7000 \mathrm{~m}$.

polynomial approximation and function substitution, the nonlinear model, including the terms, $\mathcal{A}, \mathcal{Q}$, and $\mathcal{V}$, can be written in the following form

$$
\dot{\tilde{x}}=\tilde{A}(\tilde{V}, \tilde{\alpha}) \tilde{x}+\tilde{B}(\tilde{V}, \tilde{\alpha}) \tilde{u} .
$$

The time responses of the open-loop high fidelity nonlinear (HNL), low fidelity nonlinear (LNL) and polynomial (PN) models under elevator input are compared in the following plots (Fig. 3). The elevator inputs are 1 degree when $0 \leq t<10 \mathrm{~s},-1$ degree when $10 \leq t<20 \mathrm{~s}, 1$ degree when $20 \leq t<40 \mathrm{~s}$ and 0 when $t \geq 40 \mathrm{~s}$. The plots show the deviations of the states of open-loop models from the trim states of the LNL model. The time responses of LNL model and PN model are indistinguishable. The time responses of the closed-loop HNL, LNL and PN models under $\gamma$ and $V$ commands (without elevator faults and thrust faults) are compared in Fig. 4. The plots of $\tilde{V}$, $\tilde{\gamma}$ of the HNL, LNL and PN models almost overlap. Also shown in the plots are velocity command (cmd) and $\gamma$ command. Note that there is a small difference in the phugoid period due to the polynomial fitting of the aerodynamic coefficients and the approximation of the nonlinear terms. Overall, the plots show that the developed PN model is useful for later robustness analysis of the integrated closed-loop system. The LNL model is just a middle step to obtain the PN model.

\subsection{FDI filters}

This section briefly describes the LPV-FDI filters for the longitudinal motion of a Boeing 747-100/200 in [26]. The elevators of the airplane are lumped together as one actuator, and all four engines are considered as another actuator, as assumed in [26]. Reference [26] chose to design one filter for all the elevators and another filter for all engines. The equations of the FDI filters in matrix form [26] are 


$$
\begin{gathered}
\dot{w}_{i}(t)=N_{i}\left(\rho_{s c h}\right) w_{i}(t)-G_{i}\left(\rho_{s c h}\right) x(t)+F_{i}\left(\rho_{s c h}\right) u_{f}(t) \\
r_{i}(t)=M_{i} w_{i}(t)-H_{i} x(t) \\
N_{i}\left(\rho_{s c h}\right)=N_{i 0}+N_{i 1} \rho_{s c h_{1}}+\cdots+N_{i 9} \rho_{s c h_{9}} \\
G_{i}\left(\rho_{s c h}\right)=G_{i 0}+G_{i 1} \rho_{s c h_{1}}+\cdots+G_{i 9} \rho_{s c h_{9}} \\
F_{i}\left(\rho_{s c h}\right)=F_{i 0}+F_{i 1} \rho_{s c h_{1}}+\cdots+F_{i 9} \rho_{s c h_{9}}
\end{gathered}
$$

where $w_{i}$ is the state vector of filter $i, x$ is the state vector of the airplane model, $u_{f}$ consists of the desired (unfailed) actuator outputs $\left(\delta_{e}, \delta_{s}, T\right)$ (see Fig. 2) and a constant input to the filters $\left(u_{f}=\left[\delta_{e}, \delta_{s}, T, 1\right]\right)$ [26]. The index $i=1,2$ refers to the FDI filters. Filter 1 is the elevator FDI filter; filter 2 is the thrust FDI filter. Filter 1 has four states, and filter 2 has three states [26]. $N_{i j}, G_{i j}, F_{i j}, M_{i}, H_{i}, i=1,2, j=0, \ldots, 9$ are constant matrices as given in [26]. The notation $\rho_{s c h_{j}}, j=1, \ldots, 9$ denotes scheduling parameters. The scheduling parameters are defined as $\rho_{s c h_{1}}=\bar{q}$,

$\rho_{s c h_{2}}=\frac{\bar{q}}{V}, \rho_{s c h_{3}}=\frac{1}{V}, \rho_{s c h_{4}}=\theta-\alpha, \rho_{s c h_{5}}=C_{L_{\text {basic }}} \frac{\bar{q}}{V}, \rho_{s c h_{6}}=\frac{\partial C_{L}}{\partial \delta_{e}} \frac{\bar{q}}{V}, \rho_{s c h_{7}}=C_{D_{\text {mach }}} \bar{q}, \rho_{s c h_{8}}=\frac{\partial C_{m}}{\partial \delta_{e}} \bar{q}$, $\rho_{s c h_{9}}=C_{m_{\text {basic }}} \bar{q}$ in [26]. The aerodynamic coefficients are approximated as polynomial functions of $V$ and $\alpha$, so the scheduling parameters are functions of $V, \alpha$ and $\theta$ of the airplane.

If the nonlinear FDI filters are to be put in polynomial form, the same sub-problems as those of the airplane model must be solved, i.e., the polynomial fitting of the aerodynamic coefficients, the approximation of the nonlinear terms, and the untilization of the function substitution method. As with the airplane model, the trim values of $w_{i}$ must be obtained. At trim flight conditions without actuator faults, $u=u_{f}$ (see Fig. 2), $r_{i}(t)=0$ by the design of the FDI filters, the states of the FDI filters are constant, and the rates of the states of the FDI filters are zero. Let $w_{i_{t}}$ denote the trim values of the states of the $i^{t h}$ filters, let $\tilde{w}_{i}$ be the deviations from the trim values, and let $\tilde{r}_{i}(t)$ be the deviation of the output of filter $i$ from its trim value $r_{i_{t}}$. The trim values of $w_{i}$ are found by setting $x, u_{f}, \dot{w}_{i}$ to $x_{t}, u_{t}, 0$, respectively, and solving for $w_{i_{t}}$. Eq. (23) can be rewritten as

$$
\begin{aligned}
& \dot{\tilde{w}}_{i}(t)=\tilde{N}_{i}(\tilde{V}, \tilde{\alpha}) \tilde{w}_{i}(t)-\tilde{G}_{i}(\tilde{V}, \tilde{\alpha}, \tilde{\theta}) \tilde{x}(t)+\tilde{F}_{i}(\tilde{V}, \tilde{\alpha}) \tilde{u}_{f}(t) \\
& r_{i}(t)=\tilde{r}_{i}(t)+\underbrace{r_{i_{t}}}_{0}=M_{i} \tilde{w}_{i}(t)-H_{i} \tilde{x}(t)+\underbrace{M_{i} w_{i_{t}}-H_{i} x_{t}}_{0}=M_{i} \tilde{w}_{i}(t)-H_{i} \tilde{x}(t)
\end{aligned}
$$

where $\tilde{N}_{i}(\tilde{x}), \tilde{G}_{i}(\tilde{x})$ and $\tilde{F}_{i}(\tilde{x})$ are $N_{i}, G_{i}$ and $F_{i}$ after function substitution. With the assumptions of constant air mass density and constant speed of sound in the neighborhood of the considered altitude, it was noticed that the altitude of the airplane, the fourth state of the elevator FDI filter, and the third state of the thrust FDI filter were weakly coupled with other states of the FDI filters and had negligible effects on the output of the FDI filters. Those states are removed from the PN models of the FDI filters. As mentioned previously, the state removal is problem-dependent.

The time responses of the two FDI filters under elevator fault and thrust fault are presented in Fig. 5. The legend "HNL" indicates that the HNL model of the airplane and HNL model of the FDI filters are in the closed-loop system. The legend "LNL" indicates that the LNL model of the airplane and the LNL model of the FDI filters are in the closedloop system. The legend "PN" indicates that the PN models of the airplane and the FDI filters are in the closed-loop system. The simulation is started with no faults and zero initial states of the FDI filters. The dashed lines in Fig. 5 represent time-varying actuator faults. Piecewise constant elevator faults are assumed to occur at 50, 80, 120, 160, 200 and 250 seconds, and thrust faults are assumed to occur at 40, and 150 seconds. The initial conditions of the HNL FDI filters are not at trim values $[18,26]$; therefore, the time responses of the HNL filters have an initial transient. It takes about 20 seconds for both HNL filters to converge to the faults, and after that the time responses of all the models of 
the FDI filters are close to each other. It is observed that for an elevator fault of 2 degrees from $120^{\text {th }}$ second to $160^{t h}$ second, the output of the elevator FDI filter is about 2.6 degrees (see Fig. 5); that is, the elevator FDI filter has about one-degree fault estimation error per three-degree elevator fault. The observation will be used later to determine the weighting function for the performance of the elevator FDI filter.
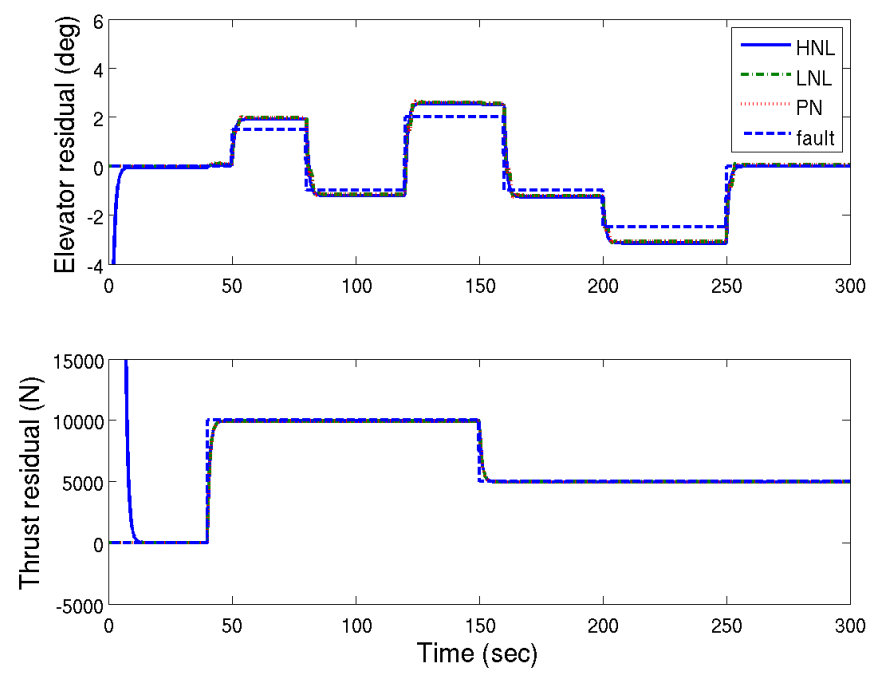

Figure 5: Time responses of the LPV-FDI filters under elevator faults and thrust faults.

\subsection{Combining the models of Boeing 747-100/200 and the FDI filters}

Rather than considering models of the airplane and the FDI filters separately, they are augmented because they have the same scheduling parameters (see Eq. (22) and Eq. (25)). The combined state vector is $\tilde{x}_{p}=\left[\tilde{x} ; \tilde{w}_{1} ; \tilde{w}_{2}\right]$, where $\tilde{x}$, $\tilde{w}_{1}, \tilde{w}_{2}$ are the states of the corresponding PN models. The combined equations of the plant and filters becomes

$$
\left\{\begin{array}{c}
\dot{\tilde{x}} \\
\dot{\tilde{w}}_{1} \\
\dot{\tilde{w}}_{2}
\end{array}\right\}=\underbrace{\left[\begin{array}{lllll}
\tilde{A}\left(\tilde{\rho}_{s y s}\right) & 0 & 0 & \tilde{B}\left(\tilde{\rho}_{s y s}\right) & 0 \\
-\tilde{G}_{1}\left(\tilde{\rho}_{\text {sys }}\right) & \tilde{N}_{1}\left(\tilde{\rho}_{s y s}\right) & 0 & 0 & \tilde{F}_{1}\left(\tilde{\rho}_{s y s}\right) \\
-\tilde{G}_{2}\left(\tilde{\rho}_{\text {sys }}\right) & 0 & \tilde{N}_{2}\left(\tilde{\rho}_{s y s}\right) & 0 & \tilde{F}_{2}\left(\tilde{\rho}_{s y s}\right)
\end{array}\right]}_{\tilde{s}}\left\{\begin{array}{c}
\tilde{x} \\
\tilde{w}_{1} \\
\tilde{w}_{2} \\
\tilde{u} \\
\tilde{u}_{f}
\end{array}\right\}
$$

$\tilde{\rho}_{\text {sys }}$ refers to $\tilde{V}$ and $\tilde{\alpha}(\tilde{\theta}$ in Eq. (25) is treated as a state variable rather than a scheduling parameter). The vectors $\tilde{u}$ and $\tilde{u}_{f}$ are inputs to the airplane model and inputs to the FDI filters, respectively. Inputs to the airplane are the actuator

outputs plus faults, if any (Fig. 2). Inputs to the FDI filters are the predicted actuator outputs. $\tilde{S}$ (see Appendix) is a polynomial matrix function of $\tilde{V}$ and $\tilde{\alpha}$. The highest degree of $\tilde{V}$ in $\tilde{S}$ is 4 and that of $\tilde{\alpha}$ is 1. Eq. (26) is expressed in 
LFT format using the matrix-based LFT modeling approach as in Eq. (1). The variation block is

$$
\Delta(\tilde{V}, \tilde{\alpha})=\operatorname{diag}\left[\tilde{V} I_{n_{V}}, \tilde{\alpha} I_{n_{\alpha}}\right]
$$

where $n_{V}=11$ and $n_{\alpha}=2$. The range of $\tilde{V}\left(=V-V_{t}\right)$ is $[-77.0,23.0] \mathrm{m} / \mathrm{s}$. The range of $\tilde{\alpha}\left(=\alpha-\alpha_{t}\right)$ is $[-0.053,0.121] \mathrm{rad}$. The LFT model is normalized based on the ranges of $\tilde{V}$ and $\tilde{\alpha}$ to obtain $\bar{\Delta}(\tilde{V}, \tilde{\alpha})$. Like $\Delta$, $\bar{\Delta}$ is not the "uncertainty" block, but represents the nonlinear variations of $\tilde{S}$ with respect to the normalized $\tilde{V}$ and normalized $\tilde{\alpha}$.

Certainly, the LFT model of the airplane and the LFT model of the LPV-FDI filters can be obtained separately and connected together to form an LFT model of the airplane and filters. However, one of the advantages of LPV-FDI filters is that their dynamics vary with the dynamics of the airplane, in this case, via $\tilde{V}$ and $\tilde{\alpha}$. Separate LFT models for the airplane and the filters lead to independent variation blocks for the airplane and the filters, and this does not utilize the strength of the LPV-FDI filters. Comparison of the analysis results of the integrated system using separate LFT models and combined LFT models will be presented in Section 4.6.

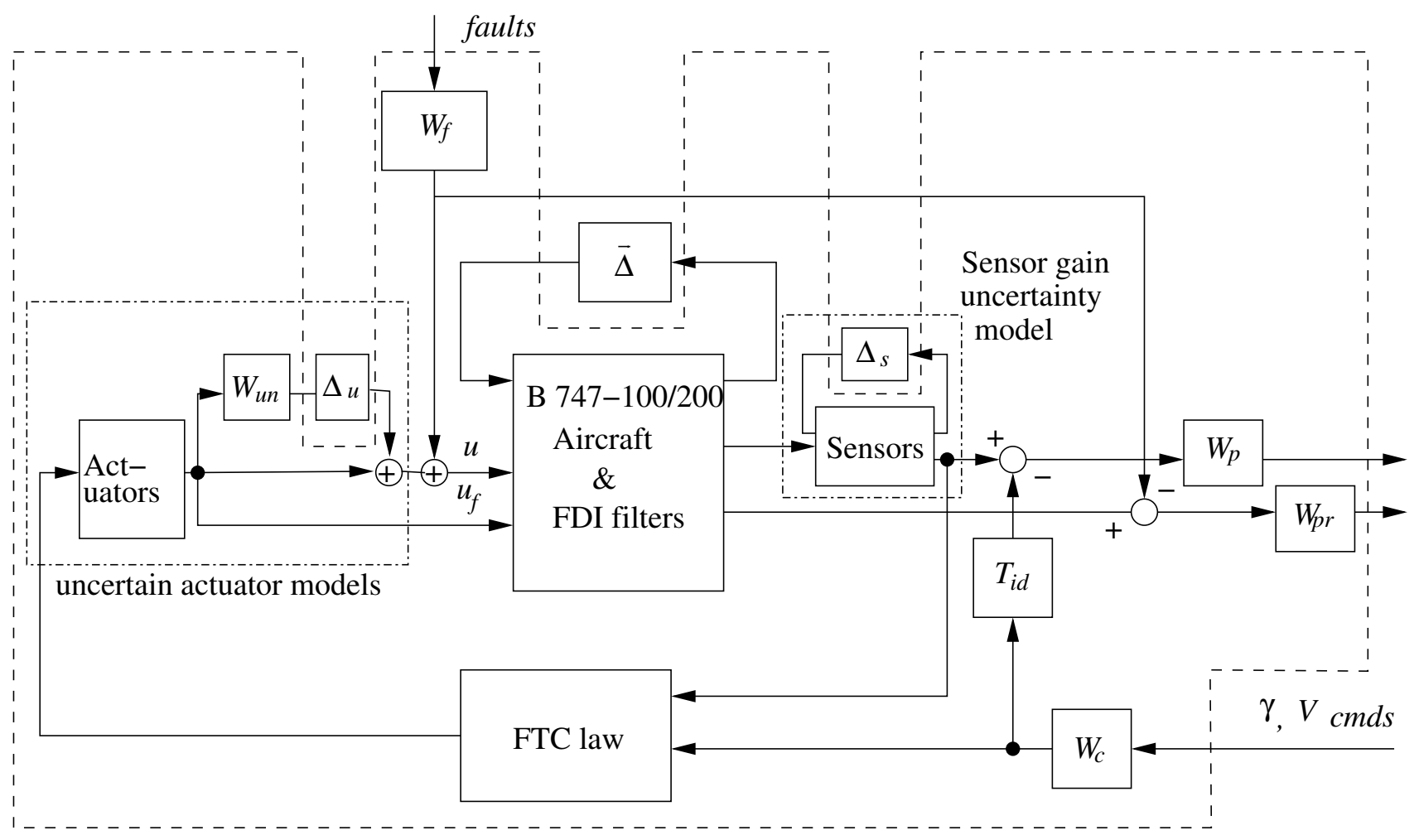

Augmented system for robustness analysis

Figure 6: Closed-loop interconnected system and weighting functions. 


\subsection{Closed-loop system with weighted performance}

The closed-loop system with weighted performance is described in Fig. 6. The block "B747-100/200 Aircraft \& FDI filters" with the feedback block " $\bar{\Delta}$ " is the normalized LFT model in Fig. 1. The transfer functions $T_{i d_{\gamma}}, T_{i d_{V}}$ and weighting functions in Fig. 6 are

$$
\begin{aligned}
& T_{i d}=\operatorname{diag}\left(T_{i d_{\gamma}}, T_{i d_{V}}\right) \\
& W_{p}=\operatorname{diag}\left(W_{p_{e}}, W_{p_{V}}\right) \\
& W_{p r}=\operatorname{diag}\left(W_{p r 1}, W_{p r 2}\right) \\
& W_{f}=\operatorname{diag}(3 \operatorname{deg}, 10000 \mathrm{~N})
\end{aligned}
$$

$$
\begin{aligned}
& T_{i d_{\gamma}}=\frac{0.35^{2}}{s^{2}+0.7 s+0.35^{2}} \\
& W_{p_{e}}=\frac{\frac{180}{\pi} 40\left(\frac{s}{100}+1\right)}{\frac{s}{0.005}+1} \\
& W_{p r 1}=\frac{0.33(0.1 s+1)^{2}}{(2 s+1)^{2}}
\end{aligned}
$$$$
T_{i d_{V}}=\frac{0.15^{2}}{s^{2}+0.3 s+0.15^{2}}
$$$$
W_{p_{V}}=\frac{100\left(\frac{s}{100}+1\right)}{\frac{s}{0.005}+1}
$$$$
W_{p r 2}=\frac{0.001(0.001 s+1)^{2}}{(10 s+1)^{2}}
$$

$T_{i d_{\gamma}}$ and $T_{i d_{V}}$, taken from [26], are the desired tracking models from $\gamma_{c m d}$ to $\gamma$ and from $V_{c m d}$ to $V$, respectively. $W_{p_{e}}$ is the weighting function to penalize the flight path angle tracking error. $W_{p_{V}}$ is the weighting function to penalize the velocity tracking error. $W_{p r 1}$ and $W_{p r 2}$ are the weighting functions on the difference between the elevator fault and the output of filter 1 and on the difference between thrust fault and the output of filter 2, respectively. $W_{p r 1}$ allows about one-degree error in the detection of three-degree elevator faults at low frequency. Recall that the output of the elevator FDI filter is about 2.6 degrees for a two-degree elevator fault (Fig. 5). Therefore, for a three-degree elevator fault, the error in the fault estimate is about 1 degree. $W_{p r 2}$ allows $1000 \mathrm{~N}$ error in the detection of $10000 \mathrm{~N}$ thrust fault at low frequency. At high frequency, $W_{p r 1}$ and $W_{p r 2}$ relax the weights on the differences. $W_{c}$ is given in Section 4.6.

\subsection{Results of robust performance analysis}

This section presents the robust performance analysis results of the integrated LPV-FDI filters and the closed-loop FTC system of a Boeing 747-100/200 at $7000 \mathrm{~m}$ over the considered flight envelope, $V=[150,250] \mathrm{m} / \mathrm{s}$ and $\alpha=[-2,8] \mathrm{deg}$. This section also compares the performance analysis by using the combined LFT model and the separate LFT models of the airplane and the LPV-FDI filters. In addition, the performance of the linearized LTI-FDI filters at the LNL trim conditions is considered. Nonlinear time simulations are performed on the HNL models to validate the $\mu$ analysis results. In this analysis, the weighting matrix block $W_{c}$ (see Fig. 6) describes the maximum size of the allowable commanding signals. Three levels of commanding signals are considered; they are (case 1) $W_{c}=\operatorname{diag}(1 \mathrm{deg}, 1 \mathrm{~m} / \mathrm{s})$, (case 2) $W_{c}=\operatorname{diag}(3 \mathrm{deg}, 5 \mathrm{~m} / \mathrm{s})$, and (case 3$) W_{c}=\operatorname{diag}(6 \mathrm{deg}, 10 \mathrm{~m} / \mathrm{s})$.

The weighted nominal performance from the four inputs, $\gamma$ command, velocity command, elevator fault and thrust fault, to the four outputs, $\gamma$, velocity, elevator residual and throttle residual, is presented in Fig. 7. The word "nominal performance" is borrowed from the robust control jargon; it means the variation block $\bar{\Delta}$ in Fig. 6 is zeroed out $(\tilde{V}=0$ and $\tilde{\alpha}=0$ ), and $\Delta_{u}$ and $\Delta_{s}$ are zeroed out. The $H_{\infty}$ norm must be less than or equal to one at any frequency. If $H_{\infty}>1$ at some frequency, the nominal system does not satisfy the defined performance criteria. In that case, further robust performance analysis is not necessary. The plot in Fig. 7 shows the nominal system performs well at the LNL trim conditions for (case 1) commanded $V$ signal of $1 \mathrm{~m} / \mathrm{s}$, commanded $\gamma$ signal of $1 \mathrm{deg}$, (case 2) commanded $V$ signal of $5 \mathrm{~m} / \mathrm{s}$, commanded $\gamma$ signal of $3 \mathrm{deg}$, and (case 3) commanded $V$ signal of $10 \mathrm{~m} / \mathrm{s}$, commanded $\gamma$ signal of 6 deg. It is observed from Fig. 7 that the nominal performance gets worse as the command signals increase. The performance of the integrated system under varying command signals was studied. Other performance analyses in this section focus on the performance of the integrated system under the same bounded command signals, but with varying uncertainty models.

Because the $H_{\infty}$ norm is less than one in the considered frequency range, the performance of the integrated LPVFDI filters and the closed-loop FTC system at any combinations of $\tilde{V}$ and $\tilde{\alpha}$ is considered. The normalized variation 


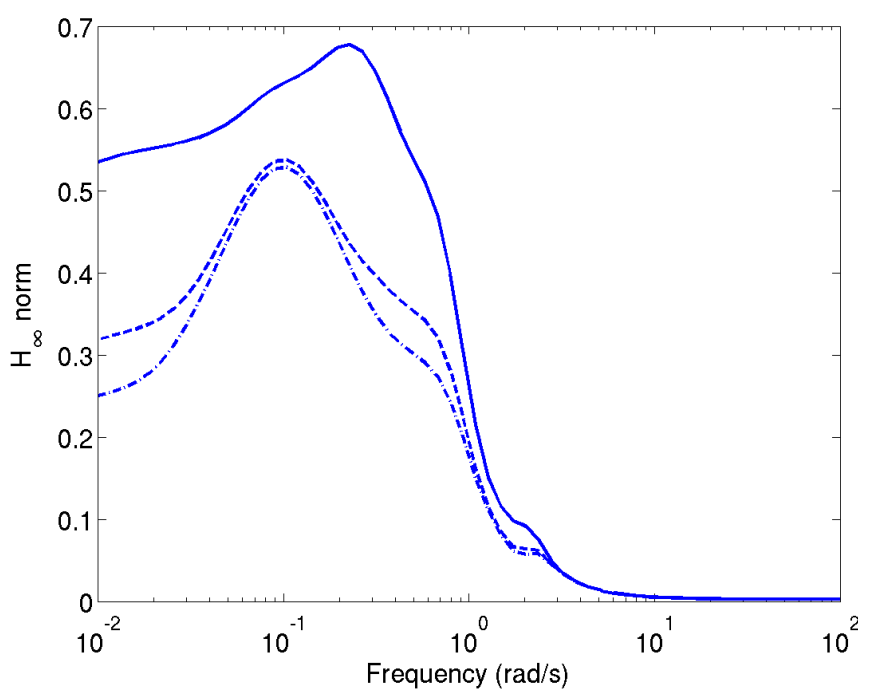

Figure 7: Nominal performance: case 1 (dash dot); case 2 (dash); case 3 (solid).
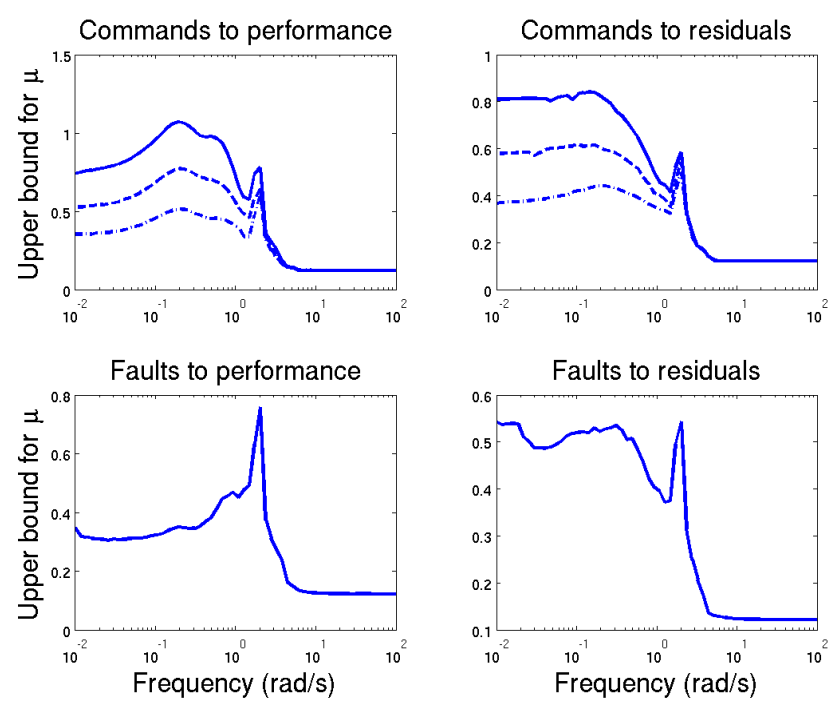

Figure 8: Performance of the system over the flight envelope without uncertainty: case 1 (dash dot), case 2 (dash), case 3 (solid).

block $\bar{\Delta}$ is non-zero, but $\Delta_{u}$ and $\Delta_{s}$ are still zeroed out. The inputs of the system, $\gamma$ command, $V$ command, elevator fault and thrust fault, and the outputs of the system, $W_{p_{e}}, W_{p_{v}}, W_{p r 1}$, and $W_{p r 2}$, are connected by fictitious full uncertainty blocks, and the plots of the upper bound for $\mu$ are presented in Fig. 8 for three cases of the command gains. The word "performance" in the titles of the sub-plots in Fig. 8 refers to the outputs of $W_{p}$ in Fig. 6. The top left subplot shows the performance of the airplane without faults, the top right subplot shows the coupling effect from the $V$ and $\gamma$ commands to the outputs of the FDI filters, the bottom left subplot shows the coupling effect from the faults to the airplane performance, and the bottom right subplot shows the performance of the FDI filters with zero command signals. From the top left subplot of Fig. 8, $\mu>1$ for case 3 reveals that the airplane cannot satisfy the performance requirements over the considered flight envelope when the commanded $V$ signal is $10 \mathrm{~m} / \mathrm{s}$, and commanded $\gamma$ signal is $6 \mathrm{deg}$. Even though the airplane performance degrades, the performance of the LPV-FDI filters is satisfactory over the considered flight envelope under the same command signals as shown in the right subplots of Fig. 8.

This method does not only reveal how the system performs at trim conditions or how the system performs if the parameters are within their ranges, but it also helps answer many questions regarding the performance of the LPVFDI filters. For example, the same method can be used to study the performance of the LPV-FDI filters over new ranges of the considered parameters ( $V$ and $\alpha$ for this particular problem), or how the filters perform with a slightly different model of the airplane, or how the filters perform when the system model has some uncertainty. Several of the above questions are considered here. It is assumed that the uncertainty of the actuator models are represented as post multiplicative uncertainty. Let $W_{u n_{e}}$ and $W_{u n_{t}}$ denote uncertainty models of the elevator actuator and throttle actuator, respectively.

The bounds for $\mu$ for case 1, shown in Fig. 9, are calculated in the presence of one percent $\tilde{V}$ and $\tilde{\alpha}$ sensor gain uncertainty (dash-dotted), $1.2 \%$ (dashed) and ten percent (solid) unmodeled actuator dynamics over the considered flight envelope. Fig. 9 shows the performance of the airplane and the LPV-FDI filters under the influence of the command signals and faults. The top right subplot and bottom left subplot of Fig. 9 reveal that there are coupling effects from the command signals to the filter residuals and from the faults to the airplane performance. Fig. 10 shows the performance of the LPV-FDI filters under the combined effect of the command signals and faults. Note that the 
$1.2 \%$ uncertainty actuator models are selected as

$$
W_{u n_{e}}=W_{u n_{t}}=\frac{0.012(s / 0.5+1)}{s / 100+1}
$$

The gain 0.012 is selected to make the upper bound for $\mu$ for the performance of the FDI filters close to one but not more than one. Eq. 29 represents the maximum unmodeled actutator dynamics that still guarantees satisfactory filter performance for case 1 over the flight envelope $(\mu<1$, see the dashed lines in Fig. 9). The dash-dotted lines $(\mu<1)$ shows that the integrated system performs well for case 1 in the presence of one percent sensor gain uncertainty. The solid lines in Fig. 9 show that the LPV-FDI filters cannot achieve the pre-defined performance requirements even for case 1 . The lower bound for $\mu$ in Fig. 10 confirms that $\mu>1$ for the integrated system with ten percent unmodeled actuator dynamics. This means that there exists a flight condition within the considered flight envelope, where the performance of the LPV-FDI filters degrades. In Fig. 10, the values of $\mu$ are less than one for one percent sensor gain uncertainty, close to one for $1.2 \%$ unmodeled actuator dynamics, and greater than one for ten percent unmodeled actuator dynamics.

The HNL dynamics of the integrated system are simulated with the LPV-FDI filters and the FTC law for selected command and fault signals (shown in Fig. 11 and 12) to validate the robustness analysis results. For case 3 without uncertainty $\left(\Delta_{u}=0, \Delta_{s}=0\right)$, velocity is commanded as a $10 \mathrm{~m} / \mathrm{s}$ increase at $50 \mathrm{~s}$ and a $10 \mathrm{~m} / \mathrm{s}$ decrease at 180 $\mathrm{s}$ with a $10 \mathrm{~m} / \mathrm{s}$ velocity command offset at $0 \mathrm{~s}$. The velocity command offset leads the aircraft to flight condition of $V=238 \mathrm{~m} / \mathrm{s}$, which is close to the boundary of the considered flight envelope. It is noted that the output of the elevator LPV-FDI filter is bounded within $1 \mathrm{deg}$, and the output of the thrust LPV-FDI filter is bounded within 1000 $\mathrm{N}$ as allowed by the weighting functions in Eq. (28). The time responses of the FDI filters (marked with the symbol $*$ in Fig. 11 and Fig. 12) show that the LPV-FDI filters achieve the pre-defined performance requirements at the flight condition close to the boundary under commanded $V$ signals of $10 \mathrm{~m} / \mathrm{s}$ and commanded $\gamma$ signals of 6 deg. The satisfactory performance of the LPV-FDI filters even for case 3 confirms the robustness analysis results in Fig. 8 . The lines with the symbol $\nabla$ show the time responses of the integrated system with $1.2 \%$ unmodeled actuator dynamics. The commanded $\gamma$ signal is 1 deg up at $20 \mathrm{~s}$ and 1 deg down at $100 \mathrm{~s}$, and the commanded $V$ signal is $1 \mathrm{~m} / \mathrm{s}$ increase at $50 \mathrm{~s}$ and $1 \mathrm{~m} / \mathrm{s}$ decrease at $180 \mathrm{~s}$ with velocity command offset of $10 \mathrm{~m} / \mathrm{s}$. The lines with the symbol $\triangle$ show the time response of the system with the same pattern of the command signals in the presence of one percent sensor gain uncertainty with the same velocity command offset. The outputs of the LPV-FDI filters, corresponding to $\mu<1$ cases, stay within bounds. The time responses of the system agrees with the robustness analysis results in Fig. 9, which predicts a satisfactory performance of the FDI filters in the presence of one percent sensor gain uncertainty or $1.2 \%$ unmodeled actuator dynamics. The lines with the symbol $\bullet$ in Fig. 11 and Fig. 12 show the time responses of the integrated system with $\gamma$ command 1 deg up at $20 \mathrm{~s}$ and $1 \mathrm{deg}$ down at $100 \mathrm{~s}$ and velocity command $1 \mathrm{~m} / \mathrm{s}$ up at $50 \mathrm{~s}$ and $1 \mathrm{~m} / \mathrm{s}$ down at $180 \mathrm{~s}$ with zero offset. In this case, the LPV-FDI filters do not satisfy the performance requirements since the time responses of the residual signals are out of bounds. Again, the nonlinear time simulation correlates to the robustness analysis results in Fig. 9. Note that all vertex points of the uncertainty blocks $\Delta_{u}$ and $\Delta_{s}$ are considered for the nonlinear simulation for $\mu<1$ cases; for $\mu>1$ cases, only one time history of the filter outputs at one vertex point is shown (see Fig. 11).

The performance of the FDI filters was considered at different flight conditions, under different command signals, for different values of $\mu$, and for different types and different levels of uncertainty. However, it is of interest to investigate how the FDI filters perform under the combined effect of sensor gain uncertainty and unmodeled actuator dynamics. The performance of the system with one percent sensor gain and one percent unmodeled actuator dynamics is shown in Fig. 13 and Fig. 14. Fig. 14 indicates that the FDI filter performs well because $\mu<1$. The outputs of the FDI filters, shown in Fig. 15, at the flight conditions, presented in Fig.16, stay within bounds. Again, the time simulation correlates to the $\mu$ analysis results.

For comparison of the separate LFT models, the $\mu$ bounds of the system using the separate LFT models are calculated and shown in Fig. 17. Note that the LPV-FDI filters allow the dynamics of the filters to vary with the 

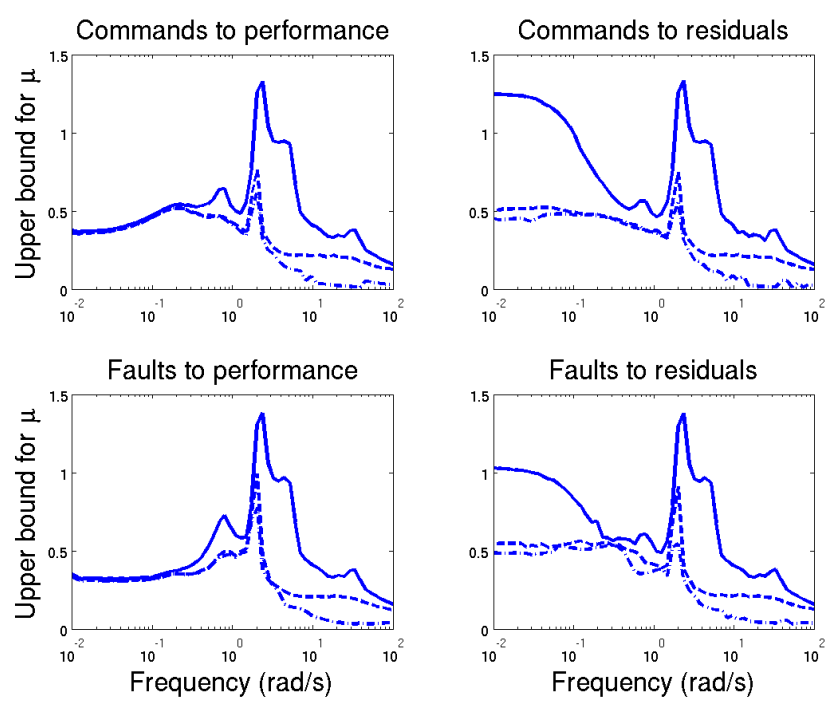

Figure 9: The upper bound for $\mu$ of the system with one percent uncertainty on the gains of the velocity sensor and the angle of attack sensor (dash dot), with $1.2 \%$ uncertainty on the actuators (dash) and with ten percent uncertainty on the actuators (solid) for case 1 .
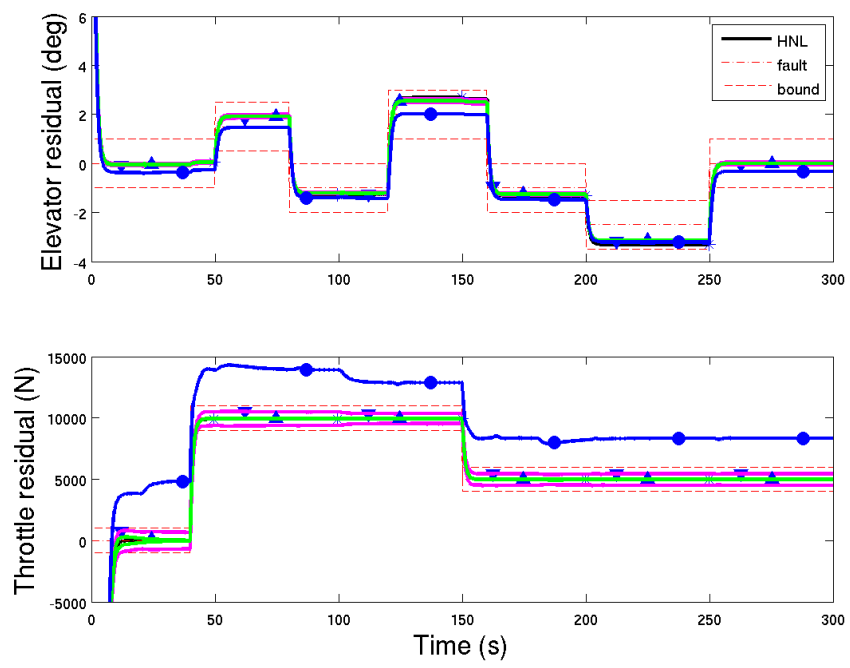

Figure 11: Time history of the LPV-FDI filters for the system with no uncertainty for case $3(*)$, with one percent uncertainty on $\tilde{V}$ and $\tilde{\alpha}$ sensor gains for case $1(\triangle)$, with $1.2 \%$ uncertainty on the actuators for case $1(\nabla)$, and with ten percent uncertainty on the actuators for case $1(\bullet)$.

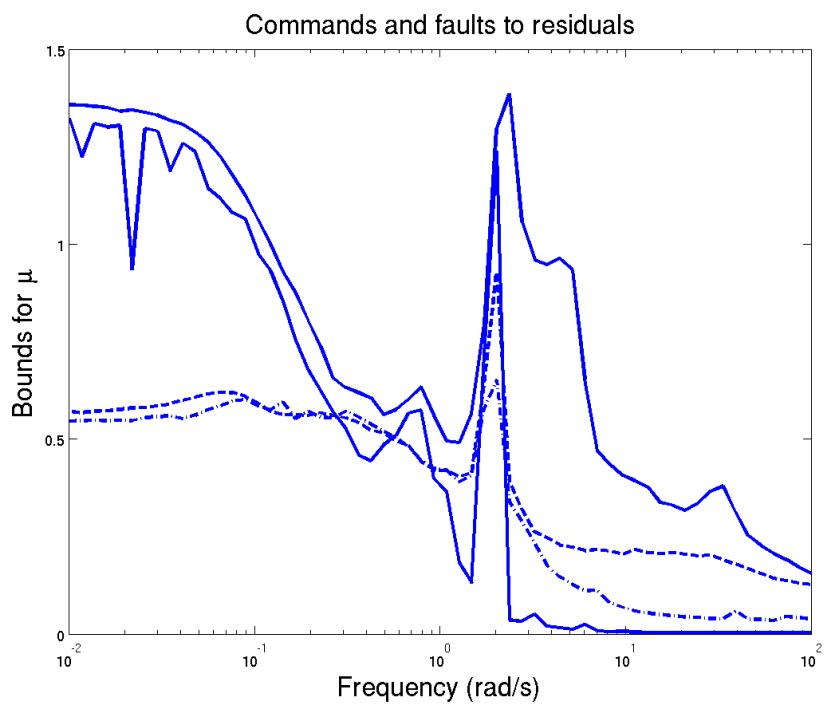

Figure 10: The upper bound for $\mu$ of the system with one percent uncertainty on the gains of the velocity sensor and the angle of attack sensor (dash dot), with $1.2 \%$ uncertainty on the actuators (dash), the lower and upper (solid) bounds for $\mu$ for the system with ten percent uncertainty on the actuators for case 1 .
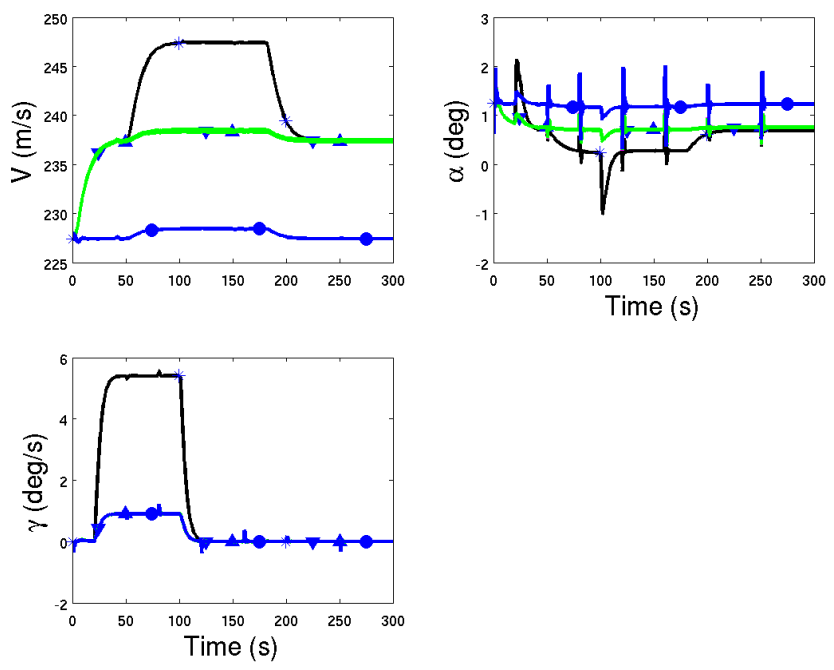

Figure 12: Time history of the closed-loop HNL system for the system with no uncertainty for case $3(*)$, with one percent uncertainty on $\tilde{V}$ and $\tilde{\alpha}$ sensor gains for case $1(\triangle)$, with $1.2 \%$ uncertainty on the actuators for case $1(\nabla)$, and with ten percent uncertainty on the actuators for case $1(\bullet)$. 

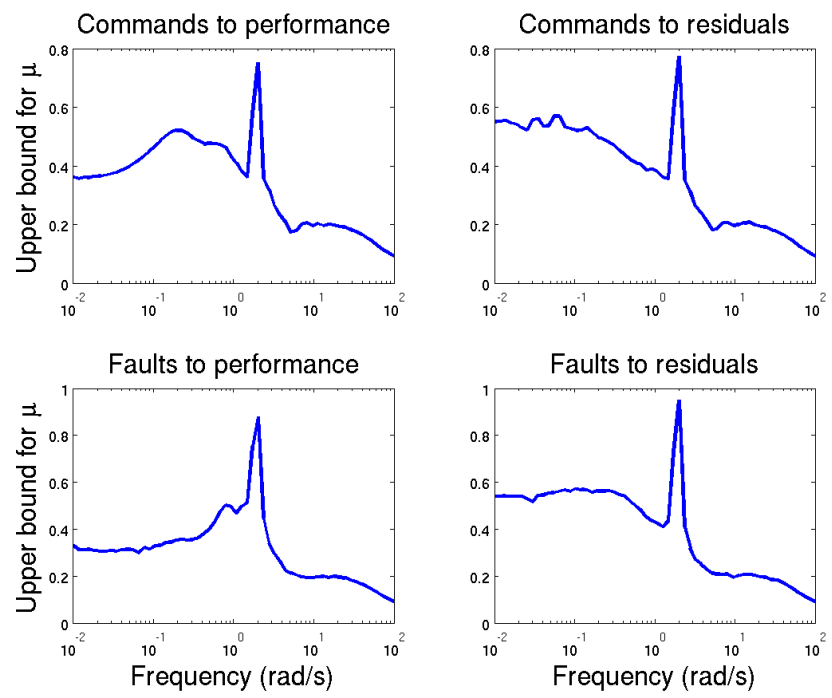

Figure 13: System with one percent uncertainty on the gains of the velocity sensor and the angle of attack sensor and one percent uncertainty on the actuators dynamics for case 1 .
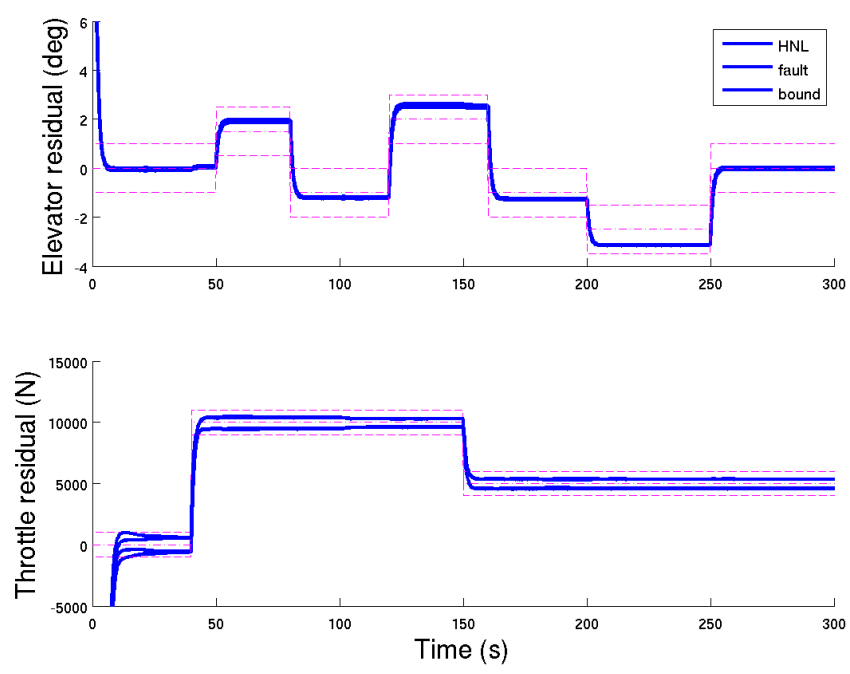

Figure 15: Time history of the LPV-FDI filters for the system with one percent uncertainty on $\tilde{V}$ and $\tilde{\alpha}$ sensor gains and one percent uncertainty on the actuators for case 1 .
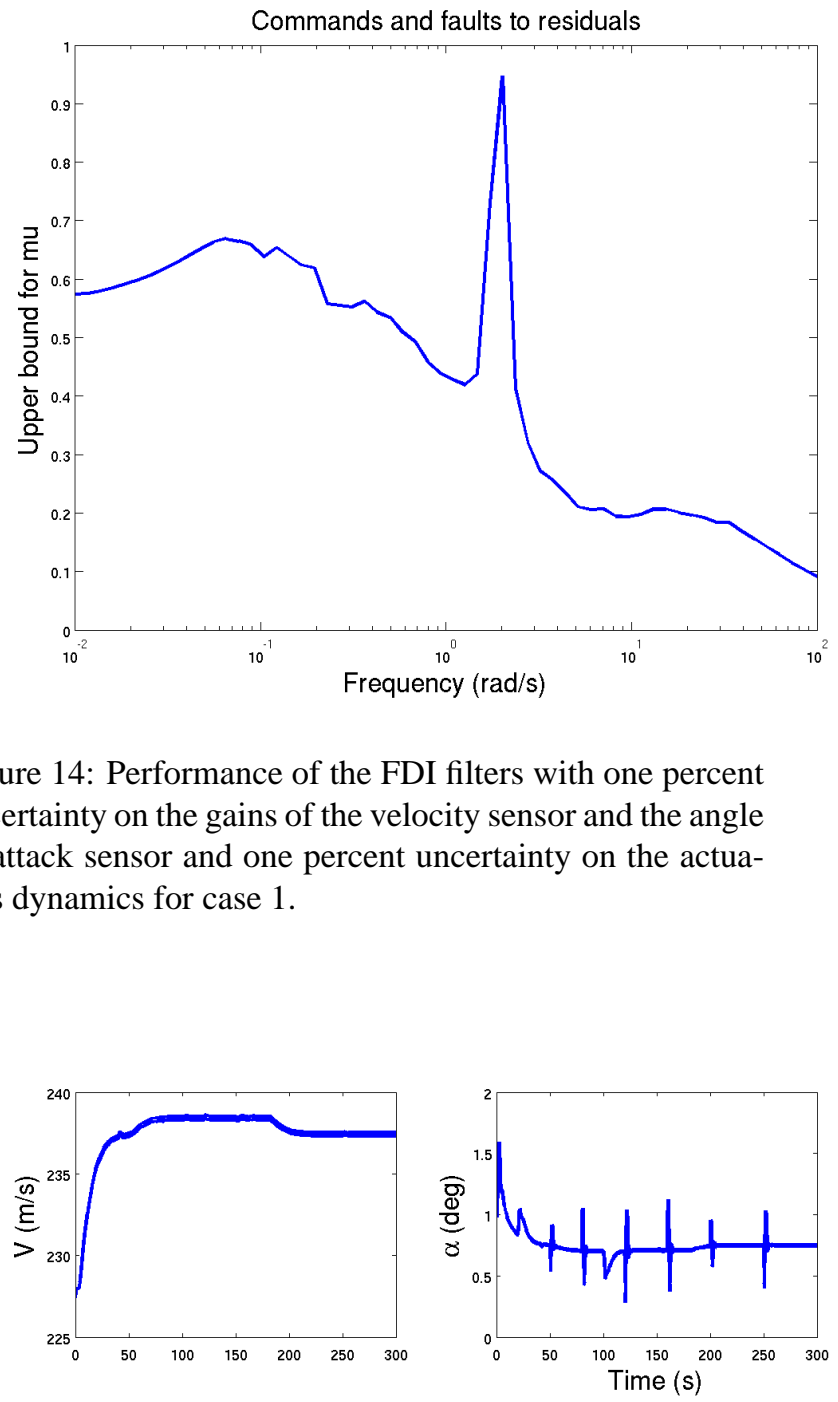

Figure 14: Performance of the FDI filters with one percent uncertainty on the gains of the velocity sensor and the angle of attack sensor and one percent uncertainty on the actuators dynamics for case 1 .

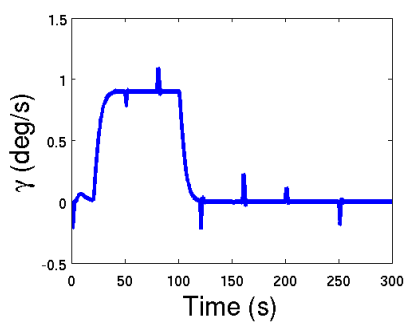

Figure 16: Time history of the closed-loop HNL system with one percent uncertainty on $\tilde{V}$ and $\tilde{\alpha}$ sensor gains and one percent uncertainty on the actuators for case 1 . 
dynamics of the model of the airplane. That is the reason why the model of the airplane and the LPV-FDI filters are combined into a plant before the LFT model is computed for the combined plant. The parameters in the variation block of the LFT model insure that the LPV-FDI filters still capture the dynamics of the airplane after the combined plant is put in LFT form. If the LFT models of the airplane and the LPV-FDI filters are found separately and then connected without combining the parameters in the variation blocks, the parameters in the variation block of one LFT model are treated by the $\mu$ analysis as independent from the parameters in the variation block of the other LFT models. That does not utilize the strength of the LPV-FDI filters because the dynamics of the LPV-FDI filters do not depend on the dynamics of the airplane. Also, the $\mu$ analysis result of the system for case 1 is more conservative, as shown in the right subplots in Fig. 17 because the parameters in each variation block are independent from parameters in the other variation blocks. The variation blocks of the components such as the aircraft dynamics, the LPV-FDI filter (filter 1) for elevator residual, and the LPV-FDI filter (filter 2) for thrust residual are $\Delta_{a / c}=\operatorname{diag}\left(\tilde{V} I_{7}, \quad \tilde{\alpha} I_{2}\right)$,

$\Delta_{f i l t e r_{1}}=\operatorname{diag}\left(\tilde{V} I_{5}, \quad \tilde{\alpha}\right)$, and $\Delta_{f i l t e r_{2}}=\operatorname{diag}\left(\tilde{V} I_{2}, \tilde{\alpha}\right)$, respectively. For $\mu$ analysis, each block is combined into

$\bar{\Delta}=\operatorname{diag}\left(\Delta_{a / c}, \quad \Delta_{\text {filter }_{1}}, \quad \Delta_{\text {filter }_{2}}\right)$. The combined block contains 14 copies of $\tilde{V}$ and 4 copies of $\tilde{\alpha}$. Recall that

the $\Delta$ block in Eq. (27) contains 11 copies of $\tilde{V}$ and 2 copies of $\tilde{\alpha}$ for the LFT model of the combined system with the LPV-FDI filters and the aircraft model. It is observed from the left subplots in Fig. 17 that the upper bounds for $\mu$ shown in the left subplots are equal to the $\mu$ values shown in the left subplots in Fig. 8 since the $\mu$ values in the left subplots are not related with the FDI filter dynamics.

For comparison of the linearized FDI filters, the lower and upper bounds for $\mu$ of the system with the linearized FDI filters are calculated for case 1 and are shown in Fig. 18. It is observed from the right subplots in Fig. 18 that the linearized FDI filters do not satisfy the pre-defined performance criteria over the flight envelope. Fig. 8 and Fig. 18 show that the LPV-FDI filters perform well in a bigger flight envelope than that covered by the linearized LTI-FDI filters.

Evaluating the performance of FDI filters with nonideal sensors (sensors with noise) and subjected to disturbance is a topic for future study. Another topic for future investigation is reconfiguration of the control laws using FDI filter outputs to make them robust in the presence of system failures. Extension of this analysis to other control methods is also of high interest.

\section{Conclusions}

The robustness analysis framework is presented for polynomial nonlinear systems using three key tools: 1) function substitution method, 2) LFT modeling approach and 3) $\mu$ analysis tool. The key of the analysis method is the LFT representation of a nonlinear multi-variable polynomial system, which can capture the nonlinear dynamic variation due to flight condition change. In the framework, it is easy to integrate with unmodeled actuator dynamics and sensor gain uncertainty. The analysis framework is applied to the robustness analysis of the integrated LPV-FDI filters and the closed-loop FTC system of a Boeing 747-100/200. Robustness analysis results are validated via nonlinear simulation in the presence of unmodeled dynamics and sensor gain uncertainty.

\section{Acknowlegement}

The authors would like to thank Christine M. Belcastro, Suresh M. Joshi, Barton J. Bacon, and Carey S. Buttrill for reviewing the abstract and the paper and for giving much valuable feedback. The second author's work is supported 

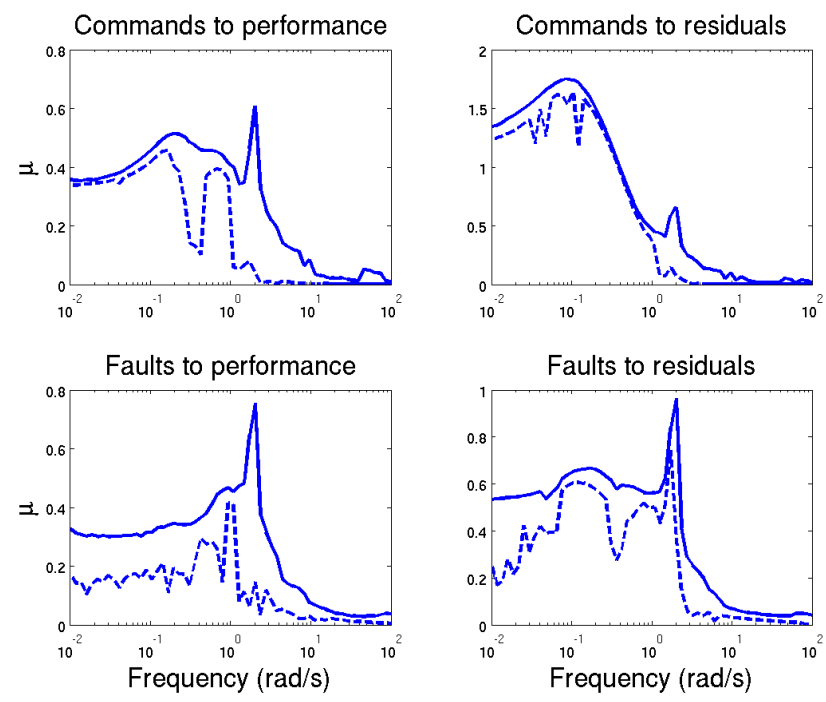

Figure 17: The lower(dash) and upper (solid) bounds for $\mu$ of the system using the separate LFT models for case 1.
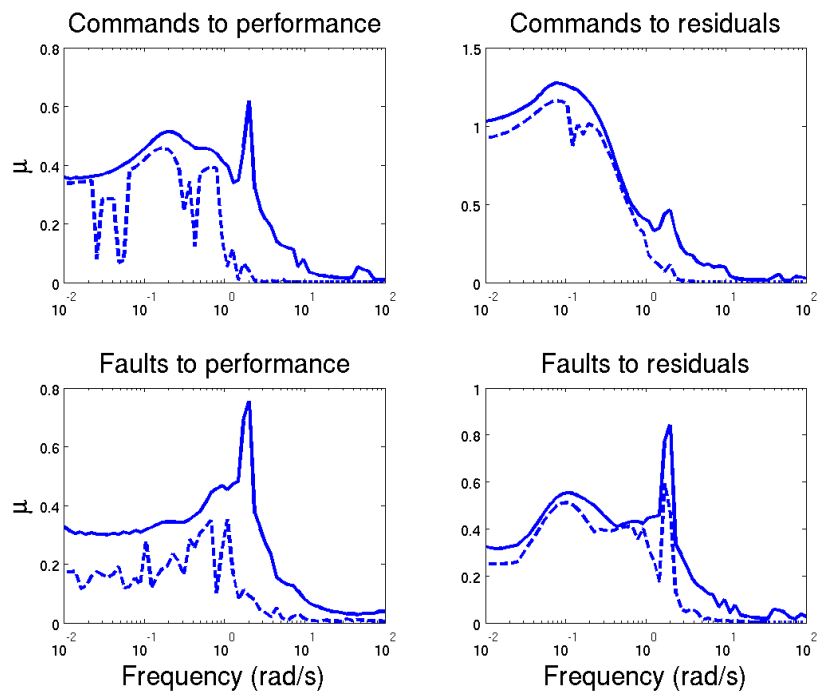

by NASA cooperative agreement \# NCC-1-02043. Thanks are also due to István Szászi, Andrés Marcos, Gary J. Balas, József Bokor and others who provideded the details of their control law, FDI filters, and Simulink simulation for Boeing 747-100/200 airplanes.

\section{Appendix: The coefficient matrix $\tilde{S}$}

Figure 18: The lower (dash) and upper (solid) bounds for $\mu$ of the system with the LTI-FDI filters for case 1.

The polynomial coefficient matrix $\tilde{S}$ with all the states of the airplane model and LPV-FDI filter models and all the control inputs are included below. Deleting the rows and columns corresponding with the altitude, the fourth state of the elevator FDI filter, the third state of the thrust filter, and the stabilizer, shifting the last three columns from the sixth row to the last row to the right, and adding zeros where appropriate yields the same $\tilde{S}$ in Eq. (26).

$$
\tilde{S}=\left[\begin{array}{rrrrrrrrrrrrrrr}
-0.59346 & 0.99143 & -0.00052124 & 0 & 0 & 0 & 0 & 0 & 0 & 0 & 0 & 0 & -0.00062513 & 0 & -3.6326 e-09 \\
-1.9626 & -0.46087 & -0.00049579 & 0 & 0 & 0 & 0 & 0 & 0 & 0 & 0 & 0 & -0.030885 & -3.9993 & 1.5328 e-07 \\
5.7733 & 0 & -0.0061168 & -9.7851 & 0 & 0 & 0 & 0 & 0 & 0 & 0 & 0 & 0 & 0 & 1.3323 e-05 \\
0 & 1 & 0 & 0 & 0 & 0 & 0 & 0 & 0 & 0 & 0 & 0 & 0 & 0 & 0 \\
-227.019 & 0 & 0 & 227.019 & 0 & 0 & 0 & 0 & 0 & 0 & 0 & 0 & 0 & 0 & 0 \\
-0.40902 & -0.94 & 0.00052087 & 0 & 0 & -1 & 0 & 0 & 0 & 0 & 0 & 0 & 0.00062513 & 0 & 4.9922 e-09 \\
-2.0291 & 0.53909 & -0.01192 & 0.11241 & 0 & 0 & -1 & 0 & 0 & 0 & 0 & 0 & -0.030883 & -3.999 & 0 \\
0 & -1 & 0 & -1 & 0 & 0 & 0 & -1 & 0 & 0 & 0 & 0 & 0 & 0 & 0 \\
-227.019 & 0 & 0 & 227.019 & 1 & 0 & 0 & 0 & -1 & 0 & 0 & 0 & 0 & 0 & 0 \\
-5.7917 & 0 & -0.99388 & 9.7789 & 0 & 0 & 0 & 0 & 0 & -1 & 0 & 0 & 0 & 0 & -1.3333 e-05 \\
0 & -1 & 0 & -1 & 0 & 0 & 0 & 0 & 0 & 0 & -1 & 0 & 0 & 0 & 0 \\
-227.019 & 0 & 0 & 227.019 & 1 & 0 & 0 & 0 & 0 & 0 & 0 & -1 & 0 & 0 & 0
\end{array}\right]
$$




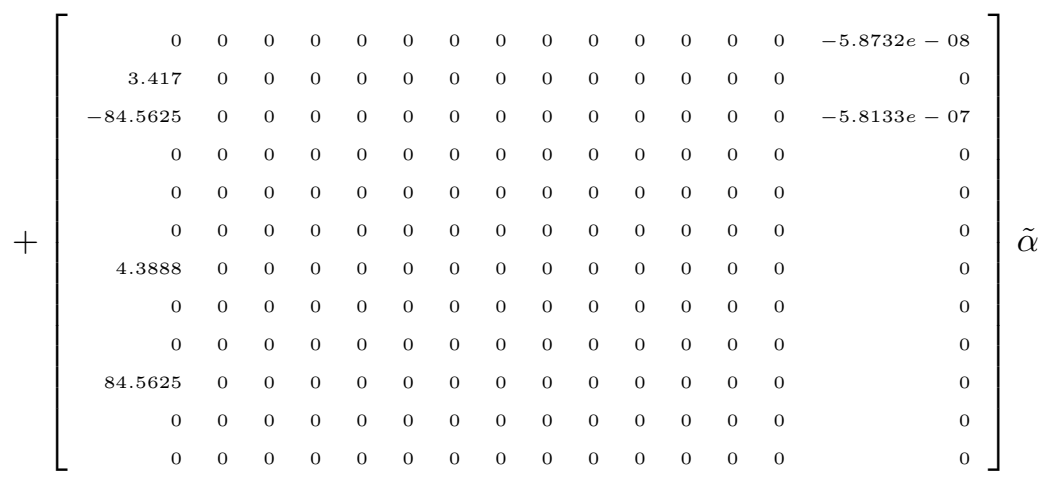

$+\quad\left[\begin{array}{rrrrrrrrrrr}-0.0025923 & 0 & -6.2678 e-07 & 0 & 0 & 0 & 0 & 0 & 0 & 0 & 0 \\ -0.01729 & -0.0020301 & -3.8893 e-06 & 0 & 0 & 0 & 0 & 0 & 0 & 0 & 0 \\ -0.035127 & 0 & -2.1091 e-05 & 0 & 0 & 0 & 0 & 0 & 0 & 0 & 0 \\ 0 & 0 & 0 & 0 & 0 & 0 & 0 & 0 & 0 & 0 & 0 \\ -1 & 0 & 0 & 1 & 0 & 0 & 0 & 0 & 0 & 0 & 0 \\ 0.0026032 & 0 & 6.2678 e-07 & 0 & 0 & 0 & 0 & 0 & 0 & 0 & 0 \\ -0.016885 & -0.00203 & -3.6466 e-06 & 0 & 0 & 0 & 0 & 0 & 0 & 0 & 0 \\ 0 & 0 & 0 & 0 & 0 & 0 & 0 & 0 & 0 & 0 & 0 \\ -1 & 0 & 0 & 1 & 0 & 0 & 0 & 0 & 0 & 0 & 0 \\ 0.035127 & 0 & 2.1091 e-05 & 0 & 0 & 0 & 0 & 0 & 0 & 0 & 0 \\ 0 & 0 & 0 & 0 & 0 & 0 & 0 & 0 & 0 & 0 & 0 \\ -1 & 0 & 0 & 1 & 0 & 0 & 0 & 0 & 0 & 0 & 0\end{array}\right.$

$\left.\begin{array}{rrrr}0 & -2.073 e-07 & 0 & 1.6002 e-11 \\ 0 & -0.00015607 & -0.035233 & 0 \\ 0 & 0 & 0 & 0 \\ 0 & 0 & 0 & 0 \\ 0 & 0 & 0 & 0 \\ 0 & 2.073 e-07 & 0 & -2.199 e-11 \\ 0 & -0.00015606 & -0.035231 & 0 \\ 0 & 0 & 0 & 0 \\ 0 & 0 & 0 & 0 \\ 0 & 0 & 0 & 0 \\ 0 & 0 & 0 & 0 \\ 0 & 0 & 0 & 0\end{array}\right] \tilde{V}$

$+\left[\begin{array}{rlllllllllllllr}0 & 0 & 0 & 0 & 0 & 0 & 0 & 0 & 0 & 0 & 0 & 0 & 0 & 0 & 2.5871 e-10 \\ 0.030103 & 0 & 0 & 0 & 0 & 0 & 0 & 0 & 0 & 0 & 0 & 0 & 0 & 0 & 0 \\ -0.74498 & 0 & 0 & 0 & 0 & 0 & 0 & 0 & 0 & 0 & 0 & 0 & 0 & 0 & 0 \\ 0 & 0 & 0 & 0 & 0 & 0 & 0 & 0 & 0 & 0 & 0 & 0 & 0 & 0 & 0 \\ 0 & 0 & 0 & 0 & 0 & 0 & 0 & 0 & 0 & 0 & 0 & 0 & 0 & 0 & 0 \\ 0 & 0 & 0 & 0 & 0 & 0 & 0 & 0 & 0 & 0 & 0 & 0 & 0 & 0 & 0 \\ 0.038665 & 0 & 0 & 0 & 0 & 0 & 0 & 0 & 0 & 0 & 0 & 0 & 0 & 0 & 0 \\ 0 & 0 & 0 & 0 & 0 & 0 & 0 & 0 & 0 & 0 & 0 & 0 & 0 & 0 & 0 \\ 0 & 0 & 0 & 0 & 0 & 0 & 0 & 0 & 0 & 0 & 0 & 0 & 0 & 0 & 0 \\ 0.74498 & 0 & 0 & 0 & 0 & 0 & 0 & 0 & 0 & 0 & 0 & 0 & 0 & 0 & 0 \\ 0 & 0 & 0 & 0 & 0 & 0 & 0 & 0 & 0 & 0 & 0 & 0 & 0 & 0 & 0\end{array}\right] \tilde{V}$ 


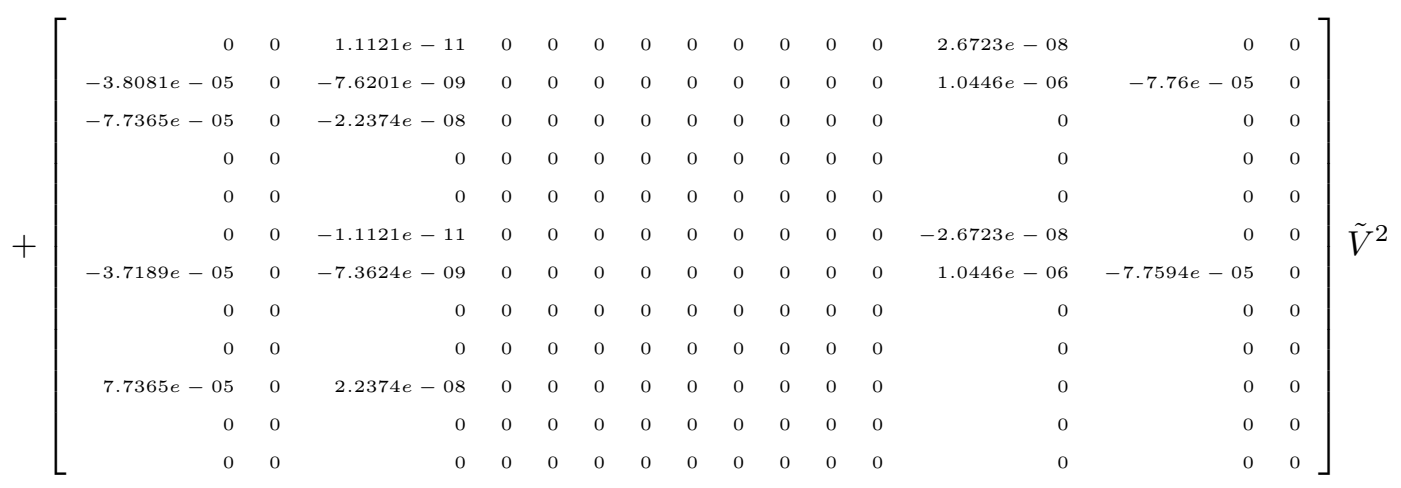

$$
+\left[\begin{array}{rllllllllllllll}
0 & 0 & 0 & 0 & 0 & 0 & 0 & 0 & 0 & 0 & 0 & 0 & 0 & 0 & 0 \\
6.63 e-05 & 0 & 0 & 0 & 0 & 0 & 0 & 0 & 0 & 0 & 0 & 0 & 0 & 0 & 0 \\
-0.0016408 & 0 & 0 & 0 & 0 & 0 & 0 & 0 & 0 & 0 & 0 & 0 & 0 & 0 & 0 \\
0 & 0 & 0 & 0 & 0 & 0 & 0 & 0 & 0 & 0 & 0 & 0 & 0 & 0 & 0 \\
0 & 0 & 0 & 0 & 0 & 0 & 0 & 0 & 0 & 0 & 0 & 0 & 0 & 0 & 0 \\
0 & 0 & 0 & 0 & 0 & 0 & 0 & 0 & 0 & 0 & 0 & 0 & 0 & 0 & 0 \\
8.5157 e-05 & 0 & 0 & 0 & 0 & 0 & 0 & 0 & 0 & 0 & 0 & 0 & 0 & 0 & 0 \\
0 & 0 & 0 & 0 & 0 & 0 & 0 & 0 & 0 & 0 & 0 & 0 & 0 & 0 & 0 \\
0 & 0 & 0 & 0 & 0 & 0 & 0 & 0 & 0 & 0 & 0 & 0 & 0 & 0 & 0 \\
0.0016408 & 0 & 0 & 0 & 0 & 0 & 0 & 0 & 0 & 0 & 0 & 0 & 0 & 0 & 0 \\
0 & 0 & 0 & 0 & 0 & 0 & 0 & 0 & 0 & 0 & 0 & 0 & 0 & 0 & 0 \\
0 & 0 & 0 & 0 & 0 & 0 & 0 & 0 & 0 & 0 & 0 & 0 & 0 & 0 & 0
\end{array}\right] \tilde{V}^{2} \tilde{\alpha}
$$$$
+\left[\begin{array}{rrrrrrrrrrrrrrr}
0 & 0 & 0 & 0 & 0 & 0 & 0 & 0 & 0 & 0 & 0 & 0 & 6.8304 e-11 & 0 & 0 \\
0 & 0 & 1.9644 e-12 & 0 & 0 & 0 & 0 & 0 & 0 & 0 & 0 & 0 & 7.7292 e-09 & 0 & 0 \\
0 & 0 & 0 & 0 & 0 & 0 & 0 & 0 & 0 & 0 & 0 & 0 & 0 & 0 & 0 \\
0 & 0 & 0 & 0 & 0 & 0 & 0 & 0 & 0 & 0 & 0 & 0 & 0 & 0 & 0 \\
0 & 0 & 0 & 0 & 0 & 0 & 0 & 0 & 0 & 0 & 0 & 0 & 0 & 0 & 0 \\
0 & 0 & 0 & 0 & 0 & 0 & 0 & 0 & 0 & 0 & 0 & 0 & -6.8304 e-11 & 0 & 0 \\
0 & 0 & 1.9643 e-12 & 0 & 0 & 0 & 0 & 0 & 0 & 0 & 0 & 0 & 7.7287 e-09 & 0 & 0 \\
0 & 0 & 0 & 0 & 0 & 0 & 0 & 0 & 0 & 0 & 0 & 0 & 0 & 0 & 0 \\
0 & 0 & 0 & 0 & 0 & 0 & 0 & 0 & 0 & 0 & 0 & 0 & 0 & 0 & 0 \\
0 & 0 & 0 & 0 & 0 & 0 & 0 & 0 & 0 & 0 & 0 & 0 & 0 & 0 & 0 \\
0 & 0 & 0 & 0 & 0 & 0 & 0 & 0 & 0 & 0 & 0 & 0 & 0 & 0 & 0 \\
0 & 0 & 0 & 0 & 0 & 0 & 0 & 0 & 0 & 0 & 0 & 0 & 0 & 0 & 0
\end{array}\right] \tilde{V}^{3}
$$

$$
+\left[\begin{array}{rrrrrrrrrrrrrrr}
0 & 0 & 0 & 0 & 0 & 0 & 0 & 0 & 0 & 0 & 0 & 0 & 0 & 0 & 0 \\
0 & 0 & 0 & 0 & 0 & 0 & 0 & 0 & 0 & 0 & 0 & 0 & 1.2065 e-11 & 0 & 0 \\
0 & 0 & 0 & 0 & 0 & 0 & 0 & 0 & 0 & 0 & 0 & 0 & 0 & 0 & 0 \\
0 & 0 & 0 & 0 & 0 & 0 & 0 & 0 & 0 & 0 & 0 & 0 & 0 & 0 & 0 \\
0 & 0 & 0 & 0 & 0 & 0 & 0 & 0 & 0 & 0 & 0 & 0 & 0 & 0 & 0 \\
0 & 0 & 0 & 0 & 0 & 0 & 0 & 0 & 0 & 0 & 0 & 0 & 0 & 0 & 0 \\
0 & 0 & 0 & 0 & 0 & 0 & 0 & 0 & 0 & 0 & 0 & 0 & 1.2065 e-11 & 0 & 0 \\
0 & 0 & 0 & 0 & 0 & 0 & 0 & 0 & 0 & 0 & 0 & 0 & 0 & 0 & 0 \\
0 & 0 & 0 & 0 & 0 & 0 & 0 & 0 & 0 & 0 & 0 & 0 & 0 & 0 & 0 \\
0 & 0 & 0 & 0 & 0 & 0 & 0 & 0 & 0 & 0 & 0 & 0 & 0 & 0 & 0 \\
0 & 0 & 0 & 0 & 0 & 0 & 0 & 0 & 0 & 0 & 0 & 0 & 0 & 0 & 0 \\
0 & 0 & 0 & 0 & 0 & 0 & 0 & 0 & 0 & 0 & 0 & 0 & 0 & 0 & 0
\end{array}\right] \underbrace{4}
$$




\section{References}

[1] Apkarian, P., P. Gahinet, and G. Becker, "Self-scheduled $H_{\infty}$ Control of Linear Parameter-Varying System: a Design Example,” Automatica, Vol. 31, No. 9, 1995, pp. 1251-1261.

[2] Balas, G., J. Doyle, K. Glover, A. Packard, and R. Smith, $\mu$-Analysis and Synthesis Toolbox For Use with Matlab, MUSYN Inc., Minneapolis, MN, 1995.

[3] Balas, G., R. Chiang, A. Packard, and M. Safonov, Robust Control Toolbox, The Mathworks, Natick, MA, 2005.

[4] Belcastro, C., "Parametric Uncertainty Modeling: An overview," Proceedings of the American Control Conference, Vol.2, Philadelphia, Pensylvania, June 1998, pp. 992-996.

[5] Belcastro, C., "On the Numerical Formulation of Parametric Linear Fractional Transformation (LFT) Uncertainty Models for Multivariate Matrix Polynomial Problems,” NASA/TM-1998-206939, November 1998.

[6] Belcastro, C., and B-C Chang, "Uncertainty Modeling for Robustness Analysis of Failure Detection and Accommodation Systems," IEEE American Control Conference, Vol. 6, American Control Conference, 2002, pp. 47764782.

[7] Belcastro, C., T. Khong, J-Y. Shin, G. Balas, H. Kwatny, and B. Chang, "Uncertainty Modeling for Robustness Analysis of Control Upset Prevention and Recovery Systems," AIAA Guidance, Navigation and Control Conference, AIAA 2005-6427, 2005, pp. 1-30.

[8] Marcos, A., A Linear Parameter Varying Model of the Boeing 747-100/200, Master thesis, University of Minnesota, 2001.

[9] Gahinet, P. and P. Apkarian, "A Linear Matrix Inequality Approach to $H_{\infty}$ Control," International Journal of Robust and Nonlinear Control, 1994, pp. 421-448.

[10] Gahinet, P., A. Nemirovski, A. Laub, and M. Chilali, LMI Control Toolbox, The Mathworks, Natick, MA, 1995.

[11] Henry, D., A. Zolghadri, "Design and analysis of robust residual generators for systems under feedback control," Automatica 41, 2005, pp. 251-264.

[12] Iwasaki, T. and G. Shibata, "LPV System Analysis via Quadratic Separator for Uncertain Implicit Systems," IEEE Transactions on Automatic Control, Vol. 46, No. 8, 2001, pp. 1195-1207.

[13] Khalil, H., Nonlinear Systems, second edition, Prentice Hall Inc., 1996.

[14] Khong, T., "Documentation on the Development of Parametric Linear Fractional Transformation (lft) Uncertainty Model Software Lft_Model $\beta$ Version,” Guidance and Control Branch, NASA Langley Research Center, 2003.

[15] Klein, V., and E. Morelli, Aircraft System Identification: Theory and Practice, AIAA Education Series, AIAA, 2006.

[16] Magni, J.-F., "Linear Fractional Representations with a Toolbox for Use with Matlab," Technical report TR 270/01 DCSD, Department of Systems Control and Flight Dynamics, December, 2001.

[17] Magni, J.-F., "Linear Fractional Representation Toolbox, Modeling, Order Reduction, Gain Scheduling," Systems Control and Flight Dynamics Department, ONERA, Toulouse Cedex, France, 2004.

[18] Marcos, A. and G. Balas, "Linear Parameter Varying Modeling of the Boeing 747-100/200 Longitudinal Motion," AIAA Guidance, Navigation and Control Conference, AIAA-01-4347, American Institute of Aeronautics and Astronautics, Montreal, Canada, Aug. 2001, pp. 1-11. 
[19] Meinsma, G., T. Iwasaki, and M. Fu, "Where Is (D,G)-Scaling Both Necessary and Sufficient," IEEE Transactions on Automatic Control, Vol. 45, No. 9, 2000, pp. 1755-1759.

[20] Morelli, E., "System Identification Programs for Aircraft (SIDPAC)," AIAA Atmospheric Flight Mechanics Conference, AIAA-2002-4704, American Institute of Aeronautics and Astronautics, Monterey, CA, August 5-8, 2002, pp. 1-18.

[21] Scherer, C., P. Gahinet, and M. Chilali, "Multiobjective Output-Feedback Control via LMI Optimization," IEEE Transactions on Automatic Control, Vol. 42, No. 7, 1997, pp. 896-903.

[22] Shin, J-Y., Worst-case Analysis and Linear Parameter Varying Control of Aerospace System, PhD thesis, Department of Aerospace Enginerring and Mechanics, University of Minnesota, 2000.

[23] Shin, J-Y, C. Belcastro, and T. Khong, "Closed-Loop Evaluation of Integrated Failure Identification for A Boeing 747-100/200 Aircraft," AIAA Guidance, Navigation and Control Conference, AIAA 2006-6310, 2006, pp. 1-22.

[24] Shin, J-Y., and I. Gregory, "Robust Gain-Scheduled Fault Tolerant Control for a Transport Aircraft," IEEE International Conference on Control Applications, 2007. Accepted.

[25] Skogestad, S. and I. Postlethwaite, Multivariable Feedback Control: Analysis and Design, John Wiley \& Sons Ltd, Baffins Lane, Chichester, 1997.

[26] Szaszi, I., A. Marcos, G. Balas, and J. Bokor, "Linear Parameter-Varying Detection Filter Design for a Boeing 747-100/200 Aircraft," Journal of Guidance, Control, and Dynamics, Vol. 28, No. 3, 2005, pp. 461-470.

[27] Tan, W., "Applications of Linear Parameter-Varying Control Theory," Master's thesis, Department of Mechanical Engineering, University of California, Berkeley, 1997.

[28] Wu, F., Control of Linear Parameter Varying Systems, Ph.D. thesis, Department of Mechanical Engineering, University of California, Berkeley, 1995.

[29] Zolghadri, A., F. Castang, and D. Henry, "Design of robust fault detection filters for multivariable feedback systems," International Journal of Modelling and Simulation, Vol. 26, No. 1, 2006, pp. 17-26. 